\title{
Shikonin blocks human lung adenocarcinoma cell migration and invasion in the inflammatory microenvironment via the IL-6/STAT3 signaling pathway
}

\author{
TAO PAN ${ }^{1-3^{*}}$, FANG ZHANG ${ }^{1,2^{*}}$, FAKAI LI $^{1}$, XINGCHUN GAO ${ }^{3}$, ZHIKUI LI $^{1}$, XIA LI $^{2}$ and XINLING REN ${ }^{1,4,5}$ \\ ${ }^{1}$ Department of Respiratory Medicine, Xijing Hospital; ${ }^{2}$ The State Key Laboratory of Cancer Biology, \\ Department of Biochemistry and Molecular Biology, The Fourth Military Medical University, Xi'an, Shaanxi 710032; \\ ${ }^{3}$ Shaanxi Key Laboratory of Brain Disorders and Institute of Basic Medical Sciences and Institute of Basic and \\ Translational Medicine, Xi'an Medical University, Xi'an, Shaanxi 710021; ${ }^{4}$ Carson International Cancer Center, \\ Shenzhen University, Shenzhen 518055; ${ }^{5}$ Shenzhen University Clinical Medical Academy, Shenzhen 518060, P.R. China
}

Received June 7, 2019; Accepted February 28, 2020

DOI: $10.3892 /$ or.2020.7683

\begin{abstract}
Increasing evidence indicates that the inflammatory tumor microenvironment can lead to cancer cell metastasis. Shikonin, which is extracted from the Chinese herb Zicao (the dried root of Lithospermum erythrorhizon), possesses various pharmacological effects, but its effect on tumor metastasis in the inflammatory microenvironment remains unknown. In the present study, we aimed to investigate the potential effect of shikonin on tumor metastasis in an inflammatory microenvironment as well as the underlying molecular mechanisms. It was found that, in the inflammatory microenvironment simulated by THP-1 cell conditioned medium (THP-1-CM) in vitro, shikonin significantly inhibited the epithelial-mesenchymal transition (EMT), migration and invasion of human lung adenocarcinoma cell lines A549 and H1299. In addition, we found that interleukin-6 (IL-6), which is expressed in THP-1-CM, promoted the EMT of lung adenocarcinoma cells, and shikonin markedly inhibited IL-6-induced EMT and cell motility. Moreover, shikonin inhibited IL-6-induced phosphorylation of signal transducer and activator of transcription 3
\end{abstract}

Correspondence to: Professor Xinling Ren, Department of Respiratory Medicine, Xijing Hospital, The Fourth Military Medical University, 169 Changle West Road, Xi'an, Shaanxi 710032, P.R. China

E-mail: majrenxl@fmmu.edu.cn

Professor Xia Li, The State Key Laboratory of Cancer Biology, Department of Biochemistry and Molecular Biology, The Fourth Military Medical University, 169 Changle West Road, Xi'an, Shaanxi 710032, P.R. China

E-mail: lixia@fmmu.edu.cn

*Contributed equally

Key words: shikonin, inflammatory microenvironment, IL-6/STAT3 signaling pathway, human lung adenocarcinoma
(STAT3), prevented phosphorylated STAT3 (p-STAT3) translocation into the nucleus, and suppressed p-STAT3 transactivation activity. Additionally, it was found that shikonin inhibited lung metastasis, EMT and expression of p-STAT3 of A549 cells in vivo. Furthermore, IL-6 levels in human lung adenocarcinoma tissues were significantly associated with tumor-node-metastasis stage and lymph node metastasis, and its expression was correlated with tumor-associated macrophage (TAM) infiltration. Together, these results suggest that shikonin suppresses the migration and invasion of human lung adenocarcinoma cells in an inflammatory microenvironment involving the IL-6/STAT3 signaling pathway.

\section{Introduction}

Lung cancer is the leading cause of cancer-related death worldwide, accounting for more than 1.5 million deaths annually (1). Adenocarcinoma, which surpasses squamous cell carcinoma as the most frequent type of lung cancer, is prone to malignant metastasis even at the early stages (2). Despite improvements in treatment for lung cancer, distant metastasis and cancer recurrence are still the main causes of death in patients with lung adenocarcinoma (3). Therefore, it is essential to develop novel treatment strategies for preventing the metastasis of lung adenocarcinoma.

Tumor metastasis is a multi-step process that involves local invasion, intravasation, extravasation and colonization, during which the formation of secondary tumors result from the spreading of primary tumor cells to a distant site $(4,5)$. The first critical step in tumor metastasis is invasion, which often requires the loss of intercellular connections and acquisition of cell motility in tumor epithelial cells (6). Epithelial-mesenchymal transition (EMT), as characterized by a loss of cell-cell adhesion, polarity and epithelial markers, is well recognized to play a crucial role in tumor invasion (5). EMT occurs during embryonic development and tissue organization under normal physiological conditions; however, its dysregulation can interrupt epithelial homeostasis and lead to various pathological conditions, including tumor metastasis (7). 
A number of clinical and experimental studies have demonstrated that an inflammatory microenvironment may contribute to tumor development and metastasis (8). Inflammatory cytokines, such as interleukin-6 (IL-6), tumor necrosis factor- $\alpha$ (TNF- $\alpha$ ), interferon- $\gamma$ and IL-1 $\beta$, are important components in an inflammatory microenvironment $(9,10)$. Among them, IL-6 is a key inflammatory factor involved in the regulation of tumorigenesis, progression and metastasis (11-13). IL-6 can activate Janus kinase (JAK)/signal transducer and activator of transcription 3 (STAT3) signaling pathway by binding to the IL-6 receptor (IL-6R) complex, which consists of IL-6R and gp130 (14). Phosphorylated STAT3 (p-STAT3; Y705) monomers can form dimers and subsequently translocate into the nucleus, in order to regulate the transcription of their target genes and eventually change the fundamental cellular processes $(15,16)$. It has been confirmed that the STAT3 signaling pathway can facilitate the EMT process by regulating the expression levels EMT-associated genes, including E-cadherin, Snail and Twist $(6,17)$.

The naphthoquinone shikonin is the main active ingredient of Zicao, a traditional Chinese herbal medicine made from the root of Lithospermum erythrorhizon (18). Shikonin has a wide spectrum of pharmacological effects as well as favorable pharmacokinetic properties in vivo (19). A number of studies have demonstrated that shikonin possesses strong antitumor activity though the inhibition of tumor growth, metastasis and angiogenesis in various cancer types $(19,20)$. Shikonin can also modulate cancer cell metabolism by inhibiting tumor-specific pyruvate kinase-M2 (21). In addition, shikonin can promote cell cycle arrest, induce necrosis and exacerbate necroptosis in certain types of tumors $(22,23)$. Furthermore, shikonin exerts different pharmacological effects in an inflammatory microenvironment (19). Considering the close association between the inflammatory microenvironment and tumor metastasis, one of its effects may be related to tumor inhibition (24). However, the potential antitumor effects of shikonin in the inflammatory microenvironment and its underlying molecular mechanisms remain largely unknown. The present study investigated the effects of shikonin on tumor metastasis and its underlying molecular mechanisms in an inflammatory microenvironment. The findings of this study could provide new insights into the mechanisms underlying the therapeutic effects of shikonin in treating inflammation-related tumor metastasis.

\section{Materials and methods}

Reagents. Shikonin was obtained from MedchemExpress. Lipopolysaccharide (LPS) and diamidino-phenyl-indole (DAPI) were supplied by Sigma-Aldrich (a brand of Merck KGaA). Recombinant human IL- 6 and TNF- $\alpha$ were obtained from PeproTech. Human IL-6 neutralizing antibody (MAB206) and human TNF- $\alpha$ neutralizing antibody (MAB610) were obtained from R\&D Systems. Bovine serum albumin (BSA) was purchased from Roche. All other reagents used in this study were of analytical grade.

Cell culture. Two human lung adenocarcinoma cell lines (A549 and H1299) and a human acute monocytic leukemia cell line (THP-1) were purchased from the Cell Bank of the Type Culture Collection of the Chinese Academy of Sciences
(Shanghai, China). Cells were cultured with F-12 medium (A549 cells; Gibco; Thermo Fisher Scientific, Inc.) and RPMI-1640 medium (H1299 and THP-1 cells; Gibco; Thermo Fisher Scientific, Inc.) containing 10\% fetal bovine serum (FBS) and maintained in a humidified atmosphere of $95 \%$ air and $5 \% \mathrm{CO}_{2}$ at $37^{\circ} \mathrm{C}$.

THP-1 cell conditioned medium. THP-1 cell conditioned medium (THP-1-CM) was prepared as previously described (6). Briefly, after adding $10 \mu \mathrm{g} / \mathrm{ml}$ LPS into THP-1 cells $\left(1 \times 10^{6}\right.$ cells $\left./ \mathrm{ml}\right)$ for $24 \mathrm{~h}$, the supernatant was collected by centrifuging at $1,520 \mathrm{x} \mathrm{g}$ for $15 \mathrm{~min}$. Then, A549 and H1299 cells were treated with THP-1-CM and different concentrations $(0.25,0.75$ or $1.25 \mu \mathrm{M})$ of shikonin for $24 \mathrm{~h}$.

Cell viability assay. Cell viability was determined using a Cell Counting Kit-8 (CCK-8) assay (Beyotime Institute of Biotechnology). Briefly, the cells (A549 and H1299) were seeded into 96 -well plates at $5 \times 10^{3}$ cells/well and incubated under normal culture conditions for $24 \mathrm{~h}$, and then the cells were treated with the indicated concentrations of shikonin and/or THP-1-CM for another $24 \mathrm{~h}$. After treatment, $10 \mu \mathrm{l}$ of CCK- 8 solution was added into each well of the 96-well plate (total medium $100 \mu \mathrm{l} /$ well) and incubated for $1 \mathrm{~h}$ at $37^{\circ} \mathrm{C}$. Optical density values were detected using a microplate reader (Model 550, Bio-Rad Laboratories) at $450 \mathrm{~nm}$. The experiment was independently repeated three times with five replicates.

Wound healing assay. The migration ability of THP-1-CMtreated lung adenocarcinoma cells (A549 and H1299) was evaluated using a wound healing assay. Briefly, the cells were seeded in a 6 -well plate at $5 \times 10^{5}$ cells/well and allowed to grow up to $80 \%$ confluence. Subsequently, the cell monolayer was scratched with a pipette tip to create a narrow wound-like gap. Shortly after wounding, the cells were washed with phosphate-buffered saline (PBS) and further treated with various concentrations of shikonin and/or THP-1-CM for $24 \mathrm{~h}$. The plates were photographed at 0 and $24 \mathrm{~h}$ with an inverted light microscope (IX53 Olympus; magnification $\mathrm{x} 40$ ). The relative migrated distance was then analyzed. The experiment was independently repeated three times with three replicates.

Transwell chamber migration and invasion assays. Chamber migration and invasion assays were performed using a Transwell assay system (Corning Costar) as reported previously $(25,26)$. After treatment under the indicated experimental conditions, the cells (A549 and H1299, 1x10 cells/chamber) suspended in $100 \mu 1$ serum-free medium were added to the upper chamber, while the lower chamber was filled with complete medium containing $10 \%$ serum. The cells were allowed to migrate at $37^{\circ} \mathrm{C}$ for $24 \mathrm{~h}$. After removing non-migrated cells, the membranes were fixed with $4 \%$ formaldehyde for $20 \mathrm{~min}$. At the end of fixation, the chambers were rinsed with PBS, and the cells in the lower chamber were stained with $0.5 \%$ crystal violet (Beyotime Institute of Biotechnology) for $10 \mathrm{~min}$. Migrated cells were quantified in five random fields per membrane, and each group was assayed in triplicate. The invasion assay was performed as per the migration assay, except that the filter membrane was coated with Matrigel (BD Bioscience). The experiment was repeated three times independently. 
Western blotting. The cells (A549 and H1299) were seeded in 6-well plates at $5 \times 10^{5}$ cells/well and allowed to grow up to $\sim 70 \%$ confluence under normal culture conditions, then the cells were treated with indicated administration conditions for $24 \mathrm{~h}$. After treatment, the total protein was isolated from cells by RIPA buffer, and the concentrations of proteins were determined using a bicinchoninic acid protein assay kit according to the manufacturer's protocol (Beyotime Institute of Biotechnology). Equal amounts of protein (30 $\mu \mathrm{g}$ protein per lane) from each sample were separated by $10 \%$ SDS-PAGE and transferred to nitrocellulose filter membrane at $100 \mathrm{mV}$ for $75 \mathrm{~min}$. After being blocked with $5 \%$ non-fat skim milk [diluted with Tris-buffered saline containing $0.1 \%$ Tween 20 (TBST)] for $1 \mathrm{~h}$ at room temperature, the membrane was incubated overnight with primary antibody (diluted with $2 \%$ bovine serum albumin in TBST) at $4^{\circ} \mathrm{C}$. The following primary antibodies were used: anti-E-cadherin antibody (dilution 1:1,000; cat. no. ab40772; Abcam), anti-N-cadherin antibody (dilution 1:1,000; cat. no. ab18203; Abcam), anti-p-STAT3 antibody (dilution 1:2,000; cat. no. ab76315; Abcam), anti-STAT3 antibody (dilution 1:2,500; cat. no. ab119352; Abcam), anti-vimentin antibody (dilution 1:300; bs-8533R; Biosynthesis Biotechnology), anti-Flag antibody (dilution 1:1,000; 20543-1-AP; Proteintech), anti-Snail antibody (dilution 1:500; 13099-1-AP; Proteintech), anti-p-JAK2 antibody (dilution 1:1,000; 3771; Cell Signaling Technology), anti-JAK2 antibody (dilution 1:1,000; 3230; Cell Signaling Technology), and $\beta$-actin (dilution 1:1,000; sc-47778; Santa Cruz Biotechnology, Inc.). On the second day, blots were washed and incubated with anti-rabbit (dilution 1:5,000; SA00001-2; Proteintech) or anti-mouse (dilution 1:5,000; SA00001-1; Proteintech) horseradish-peroxidase-conjugated secondary antibody for $1 \mathrm{~h}$. The protein bands were visualized with an enhanced chemiluminescence western blot detection kit (Pierce Biotechnology; Thermo Fisher Scientific, Inc.), and the protein expression levels were semi-quantified by densitometry using ImageJ v1.46r (National Institutes of Health) (27,28). The experiment was repeated three times independently.

Quantitative real-time PCR. RNA isolation and RT-qPCR assay were performed as previously described (29). The cells (A549 and H1299) were seeded in 6-well plates at the number of $5 \times 10^{5}$ cells/well and allowed to grow up to about $70 \%$ confluence under normal culture conditions, then the cells were treated with indicated administration conditions for $24 \mathrm{~h}$. After treatment, TRIzol reagent (Invitrogen; Thermo Fisher Scientific, Inc.) was used to isolate total RNA from the cell lines. RNA was reverse-transcribed into cDNA by AMV reverse transcriptase (Promega). Quantitative real-time PCR was carried out with SYBR Premix Ex Taq (Takara Bio Inc.). All reactions were performed in triplicate using an ABI Prism 7500 real-time PCR instrument (Applied Biosystems; Thermo Fisher Scientific, Inc.). The relative expression levels of target genes were determined after normalization to $\beta$-actin level via the $2^{-\Delta \Delta \mathrm{Cq}}$ method (30). The experiment was independently repeated three times with three replicates. The primers used in the reactions were as follows: E-cadherin (forward, 5'-CCACCAAAGTCACGCTGAAT-3' and reverse, 5'-GGA GTTGGGAAATGTGAGC-3'), N-cadherin (forward, 5'-AGC
CTGGAACATATGTGATGA-3' and reverse, 5'-CCATAA AACGTCATGGCAGTAA-3'), vimentin (forward, 5'-GGT GGACCAGCTAACCAACG-3' and reverse, 5'-GTCAAGACG TGCCAGAGACG-3'), Snail (forward, 5'-GCCTTCAACTGC AAATACTGC-3'; reverse, 5'-CTTCTTGACATCTGAGTG GGTC-3'), $\beta$-actin (forward, 5'-TGGATCAGCAAGCAGGAG TA-3' and reverse, 5'-TCGGCCACATTGTGAACTTT-3').

Immunofluorescence. The immunofluorescence assay was performed as previously described (31). Briefly, the cells (A549 and H1299) were seeded on glass coverslips in the special dishes and allowed to grow up to about $30 \%$ confluence under normal culture conditions, then the cells were treated with indicated administration conditions for $24 \mathrm{~h}$. After treatment, the cells werefixed with $4 \%$ paraformaldehyde for 20 min, permeabilized with $0.5 \%$ Triton X-100, and blocked with $3 \%$ BSA for $1 \mathrm{~h}$. The cells were then incubated with rabbit-anti-human antibodies for E-cadherin (1:200 dilution; cat. no. 20874-1-AP; Proteintech), N-cadherin (1:200 dilution; cat. no. 13116; Cell Signaling Technology), vimentin (1:200 dilution; cat. no. 10366-1-AP; Proteintech), and p-STAT3 (1:100 dilution; cat. no. 9145S; Cell Signaling Technology) at $4^{\circ} \mathrm{C}$ overnight. After washing, the cells were incubated with FITC-conjugated goat-anti-rabbit secondary antibodies (1:200 dilution; cat. no. 31583; Invitrogen; Thermo Fisher Scientific, Inc.) for $1 \mathrm{~h}$, and the nuclei were counterstained with DAPI. Finally, the stained cells were observed using a confocal fluorescence microscope (FV1000-D, Olympus; magnification $\mathrm{x} 800)$. The mean fluorescence intensities of E-cadherin, $\mathrm{N}$-cadherin and vimentin were quantified in five random fields per sample using ImageJ v1.46r (National Institutes of Health, Bethesda) according to the specified instructions. The number of cells with p-STAT3 nuclear translocation and the corresponding total number of cells were counted in at least five random fields per sample by two independent observers in a blinded (code-marked) approach, and the percentage of cells with nuclear p-STAT3 translocation was also calculated. Each experiment was performed in triplicate.

Enzyme-linked immunosorbent assay. Both IL-6 and TNF- $\alpha$ levels in the supernatant of THP-1 cells were measured using a Quantikine enzyme-linked immunosorbent assay (ELISA) kit (R\&D Systems) according to the manufacturer's instructions. The experiment was carried out in triplicate and repeated three times independently.

Transient transfection. PcDNA3.1-Flag-STAT3 (Bioworld Technology, Inc.) was transfected into cells (A549 and H1299) using Lipofectamine $2000^{\mathrm{TM}}$ reagent (Invitrogen; Thermo Fisher Scientific, Inc.) according to the manufacturer's instructions. After $24 \mathrm{~h}$ of the transfection, the cells were treated with shikonin $(1.25 \mu \mathrm{M})$ and IL-6 $(50 \mathrm{ng} / \mathrm{ml})$ for another $24 \mathrm{~h}$, then the subsequent experiments were conducted.

Luciferase assay. Lung adenocarcinoma cells (A549 and H1299) were seeded in 48-well plates at the number of $3 \times 10^{4}$ cells/well and cultured under normal conditions for $24 \mathrm{~h}$, then transfected with $0.2 \mu \mathrm{g}$ pSTAT3-TA-luc (Beyotime Institute of Biotechnology) and $0.01 \mu \mathrm{g}$ pRL-TK Renilla (Beyotime Institute of Biotechnology) using Lipofectamine 
2000. Twenty-four hours after transfection, the cells were treated with shikonin and IL-6 $(50 \mathrm{ng} / \mathrm{ml})$ for $24 \mathrm{~h}$. After treatment, the cells were lysed using passive lysis buffer, and the luciferase activity was detected using a Dual Luciferase Reporter kit (Beyotime Institute of Biotechnology). The experiment was carried out in triplicate and repeated three times independently.

Tumor metastasis assay in vivo. All animal studies were approved by the Animal Ethics Committee of The Fourth Military Medical University (Xi'an, Shaanxi) (nos. 20150803 and 20170201). Nude mice (28-32 days old, weighing 18-24 g, $\mathrm{n}=39$ ) were obtained from Shanghai Laboratory Animal Co. and maintained in air-conditioned rooms under controlled lighting (12 h light/12 h dark). Standard laboratory water and food were available ad libitum. The animal experimental procedures were performed in strict accordance with the guidelines approved by the Institutional Review Board of Xi Jing Hospital of The Fourth Military Medical University, and conformed to the principles for the ethical use of animals.

The tumor metastasis model was established based on the experiences of our own and other studies (1,6). Briefly, A549 cells were trypsinized and resuspended in PBS to achieve an appropriate concentration $\left(1 \times 10^{6}\right.$ cells $\left./ \mathrm{ml}\right)$, and $0.2 \mathrm{ml}$ of the cell suspension was injected into the tail veins of athymic BALB/c nude mice (5). The mice were weighed and randomly divided into the following three groups ( $\mathrm{n}=5$ per group): Control group, $2.5 \mathrm{mg} / \mathrm{kg}$ shikonin group, and $5 \mathrm{mg} / \mathrm{kg}$ shikonin group (32). After $24 \mathrm{~h}$ of inoculation, shikonin was injected into the mice every other day for 24 days via intraperitoneal injection. Twenty-four hours after the last administration, the mice were euthanized with sodium pentobarbital (50 mg/kg b.w., i.p.; Sigma-Aldrich; Merck KGaA), followed by cervical dislocation. No mouse progressed to cachexia or death during the experimental period. Their lungs were rapidly excised, washed, and fixed in $4 \%$ polyoxymethylene solution. To confirm the presence of metastatic nodules as well as determine the location and extent of micrometastatic foci, the number of metastatic nodules on the entire lung surface was counted under a dissecting microscope (magnification $\mathrm{x} 20$ ) and the histological sections of lung tissue were stained routinely with hematoxylin and eosin (H\&E).

Antitumor effect in nude mice. A549 cells $\left(1 \times 10^{7}\right)$ were injected subcutaneously into the flank area of male nude mice (5). When the tumors reached an average of $75 \mathrm{~mm}^{3}$ in size, all mice were randomly divided into three groups ( $n=8$ per group). The control group was administered vehicle and the treatment groups were administered different doses of shikonin $(2.5$ or $5 \mathrm{mg} / \mathrm{kg}$ ) injected intraperitoneally every other day. After 24 days, the mice were euthanized with sodium pentobarbital (50 mg/kg b.w., i.p.; Sigma-Aldrich; Merck KGaA), followed by cervical dislocation, and their tumor xenografts were rapidly excised, weighed, and fixed in $4 \%$ paraformaldehyde for immunohistochemical analysis. The tumor volumes were measured with an electronic caliper by using the formula: Tumor volume $\left(\mathrm{mm}^{3}\right)=$ length $\mathrm{x}$ width ${ }^{2} / 2$.

Immunohistochemistry. Immunohistochemical staining was performed according to a previous description (5).
Paraformaldehyde-fixed tumor sections (4- $\mu \mathrm{m}$ in thickness) were incubated with rabbit-anti-human monoclonal antibodies p-STAT3 (1:100 dilution; product code ab76315; Abcam), E-cadherin (1:500 dilution; product code ab227639, Abcam), N-cadherin (1:150 dilution; cat. no. 13116; Cell Signaling Technology, Inc.) and vimentin (1:200 dilution; product code ab92547; Abcam), followed by incubation with horseradish peroxidase-conjugated goat-anti-rabbit secondary antibody (1:500 dilution; cat. no. WLA023; Wanleibio Co., Ltd.). The sections were then incubated with developing solution and counterstained with hematoxylin. The color was fixed with acid alcohol and dehydration steps. Finally, the sections were observed with a light microscope (magnification x100), and the representative images were photographed. The experiment was performed in triplicate.

Tissue microarray. A human lung adenocarcinoma tissue microarray (TMA) containing 128 cases of lung adenocarcinoma specimens was constructed by Xi'an Alenabio Biotechnology. The dot array was $1.5 \mathrm{~mm}$ in diameter, and each dot represented a tissue spot from one individual specimen that was specifically selected and pathologically confirmed. To compare the levels of IL-6 and CD68 in lung adenocarcinoma tissues and those in normal lung tissues and analyze the correlation between them, TMAs that composed $1.5-\mathrm{mm}$ diameter dots of 30 human lung adenocarcinoma tissues and 30 matched normal lung tissues were purchased from Outdo Biotech Co., Ltd. The study protocol was approved by the Institutional Review Board of Xi Jing Hospital of The Fourth Military Medical University. All experiments involving human subjects were performed after obtaining informed consent, and in accordance with the relevant guidelines and regulations.

Immunohistochemistry and TMA score. Immunohistochemistry of TMA was performed as described previously (33). The anti-IL-6 antibody (1:100 dilution; bs-0781R; Bioss Biological Technology) and anti-CD68 antibody (1:100 dilution; bs-0649R; Bioss Biological Technology) were used for primary antibody incubation at $4^{\circ} \mathrm{C}$ overnight, while omission of the primary antibody served as a negative control.

The immunohistochemical images of TMAs were scored by applying a semi-quantitative immunoreactivity score (IRS) as reported previously (1). Briefly, category A documented the intensity of immunostaining as 0 (negative staining), 1 (weak staining) and 2 (strong staining). Category B documented the percentage of immunoreactive cells as $1(0-25 \%), 2$ (26-50\%), $3(51-75 \%)$ and 4 (76-100\%). The IRS (range $=0-8)$ was calculated by multiplying scores from categories A and B. Samples with IRS scores of 0-3 were classified as low expression, while scores of 4-8 were classified as high expression. The results were evaluated by two independent pathologists who were blinded to the clinical data.

Statistical analysis. Statistical analyses were performed with SPSS version 16.0 software (SPSS Inc.). Data shown were obtained from at least three independent experiments. Differences between groups were compared using the Student's t-test or one-way analysis of variance (ANOVA) followed by Bonferroni's test. For TMA, the association between IL-6 

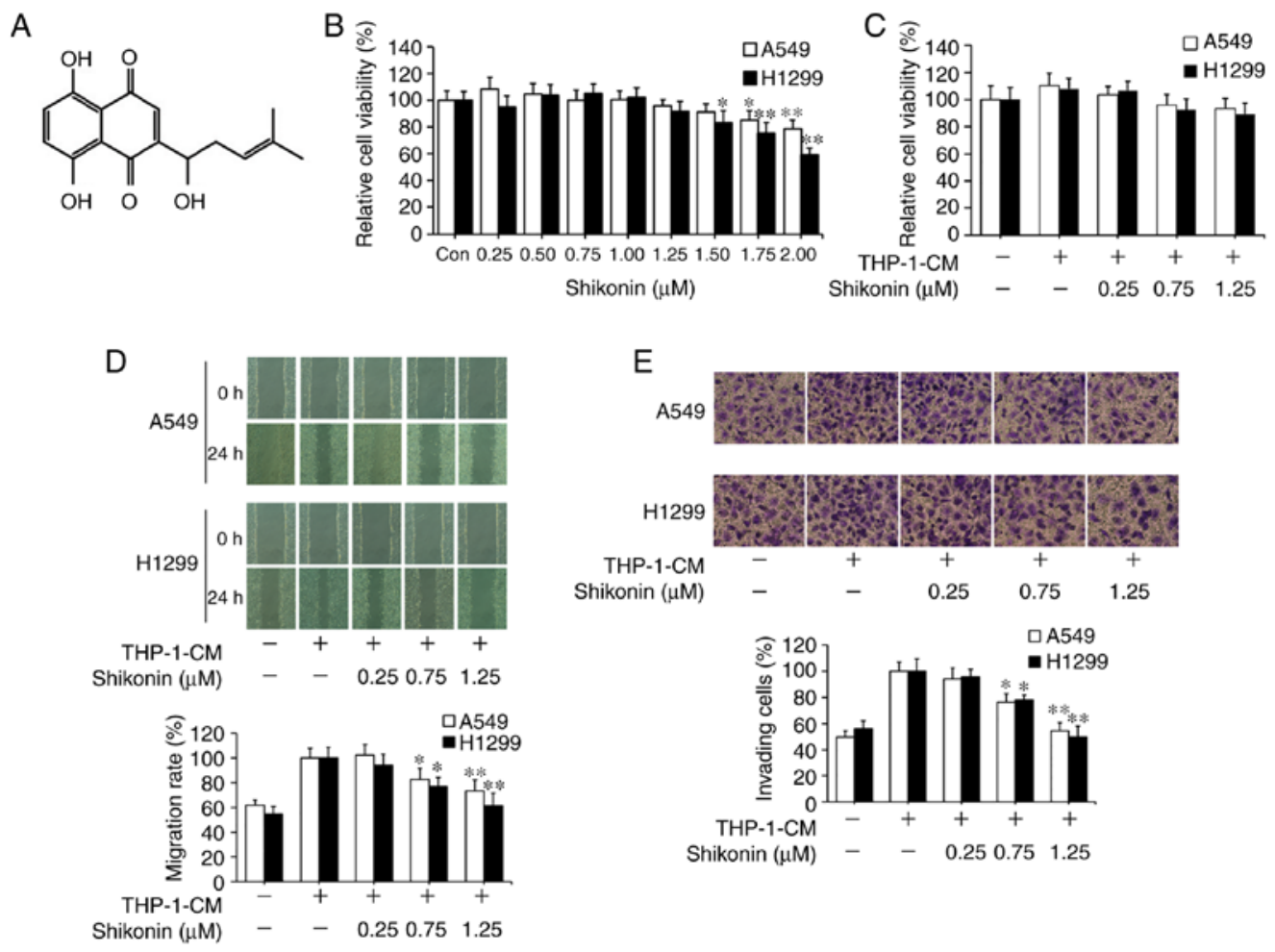

Figure 1. Inhibitory effects of shikonin on THP-1-CM-induced migration and invasion of A549 and H1299 cells. (A) Chemical structure of shikonin. (B) Effect of shikonin on the viability of lung adenocarcinoma cells. A549 and H1299 cells were treated with serial doses of shikonin in normal medium for 24 h, and the viability of cells was detected by CCK-8 assay. (C) A549 and H1299 cells were incubated with THP-1-CM and/or shikonin for 24 h, and the viability of cells was examined by CCK-8 assay. (D) Wound healing assays. A549 and H1299 cells were scratched and incubated with THP-1-CM and/or shikonin, and images were captured at 0 and $24 \mathrm{~h}$ after wounding. (E) Invasion assays were performed by Transwells covered with Matrigel. After treatment with THP-1-CM and/or shikonin for $24 \mathrm{~h}$, the cell suspensions were subjected to Transwell assays. Images (magnification x200) are representative of three independent experiments Data are shown as the mean \pm SD of three independent experiments. ${ }^{*} \mathrm{P}<0.05,{ }^{* *} \mathrm{P}<0.01$, compared with the THP-1-CM-treated group. THP-1-CM, THP-1 cell conditioned medium.

levels and clinicopathological parameters was evaluated using the Chi-square test. The correlation between IL-6 and CD68 expression was assessed using the nonparametric Spearman test. All data are expressed as mean \pm standard deviation (SD). A significant difference was defined as ${ }^{*} \mathrm{P}<0.05$ or ${ }^{* *} \mathrm{P}<0.01$ as indicated in the figures and defined in the figure legends.

\section{Results}

Shikonin blocks THP-1-CM-induced migration and invasion of human lung adenocarcinoma cells. Shikonin has been reported as a potential antitumor agent. Its chemical structure is shown in Fig. 1A. CCK-8 assay was first used to assess the effects of shikonin on lung adenocarcinoma A549 and H1299 cell viability under normal culture conditions. As shown in Fig. 1B, the cytotoxic effects of shikonin on both cell lines were in a dose-dependent manner, where no obvious cytotoxicity was observed when its concentration was equal to or less than $1.25 \mu \mathrm{M}$. Following that, THP-1-CM was used to mimic the actual inflammatory microenvironment as described previously $(5,6)$. THP-1-CM alone or in combination with $0.25,0.75$ or $1.25 \mu \mathrm{M}$ shikonin exerted no significant effect on the viability of A549 and H1299 cells (Fig. 1C). Wound healing and chamber invasion assays were used to assess the effects of shikonin on cell migration and invasion induced by THP-1-CM. As shown in Fig. 1D, upon exposure to THP-1-CM alone, elevated wound closure activity was observed in both cells lines with the scratch gap almost filled, whereas the gap still existed in both cell lines after co-treatment with THP-1-CM and shikonin $(0.75$ and $1.25 \mu \mathrm{M})$. Similarly, chamber invasion assays revealed that shikonin inhibited the invasion of A549 and H1299 cells after stimulation with THP-1-CM (Fig. 1E). These results indicate that shikonin can inhibit the migration and invasion activities of lung adenocarcinoma cells induced by THP-1-CM.

Shikonin reverses the changes in the expression levels of EMT markers induced by THP-1-CM in human lung adenocarcinoma cells. Considering that EMT can be induced by various cytokines within an inflammatory microenvironment and its deregulation may lead to tumor metastasis, the markers associated with EMT were examined in order to explore the molecular mechanism underlying the inhibitory effects of shikonin on lung adenocarcinoma cell migration and invasion activities. As shown in Fig. 2A and B, the protein expression levels of mesenchymal markers ( $\mathrm{N}$-cadherin and vimentin) were found to be increased, while the level of the epithelial marker (E-cadherin) was markedly decreased in cells cultured with THP-1-CM alone; however, such changes in expression levels were reversed by shikonin treatment. The alterations in the mRNA expression levels of E-cadherin, N-cadherin and vimentin were consistent with their protein expression levels (Fig. 2C). These results suggest that shikonin can inhibit the migration and invasion activities of lung adenocarcinoma cells by reversing THP-1-CM-induced EMT. 

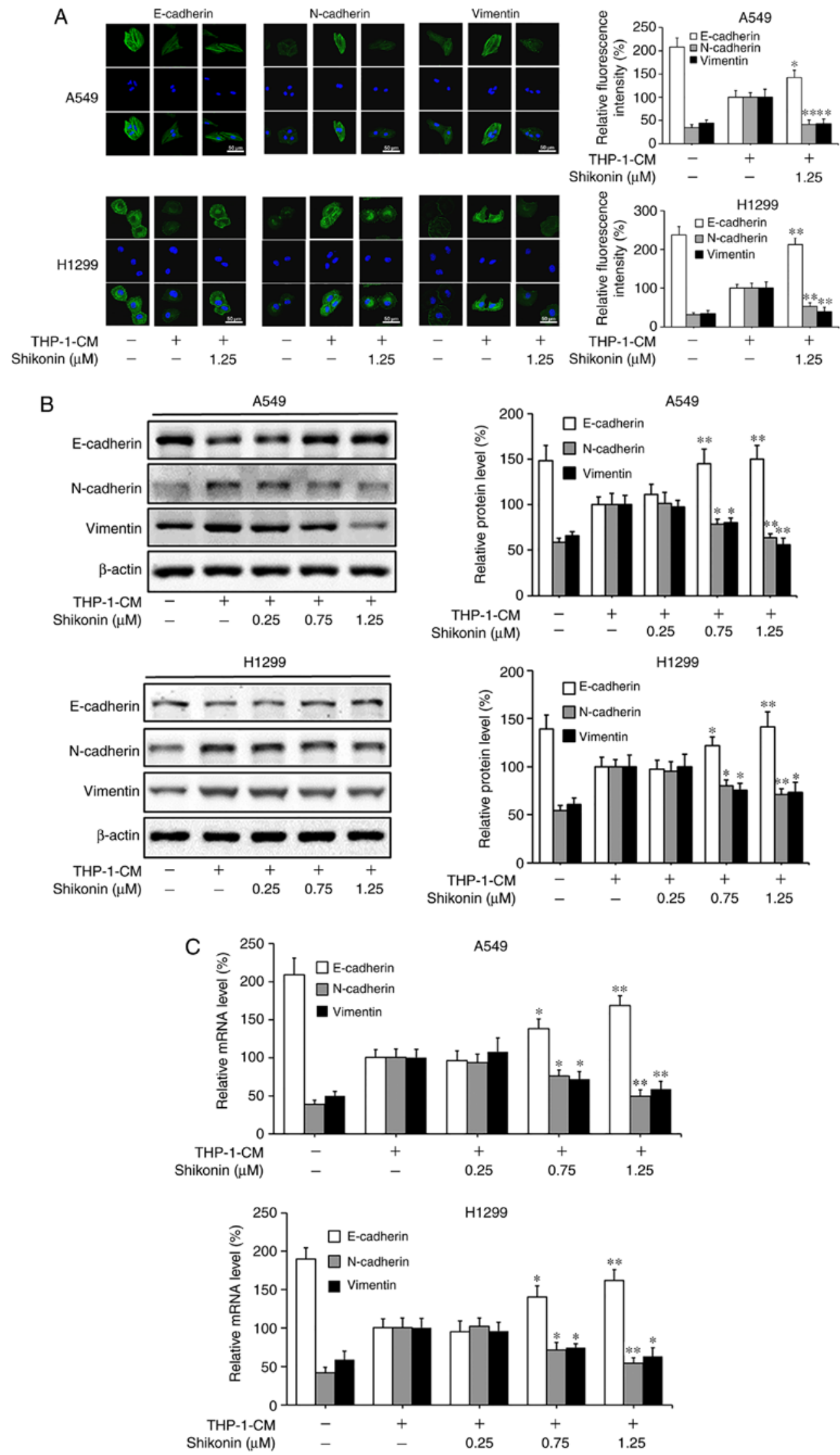

Figure 2. Shikonin suppresses THP-1-CM-induced epithelial-mesenchymal transition (EMT) in A549 and H1299 cells. (A) Immunofluorescence was used to visualize the protein expression levels of E-cadherin, $\mathrm{N}$-cadherin and vimentin after treatment with THP-1-CM and/or shikonin for $24 \mathrm{~h}$. Left, representative immunofluorescence images of E-cadherin, $\mathrm{N}$-cadherin and vimentin (magnification $\mathrm{x} 800$ ). Right, quantitative image analysis of the signal intensity of E-cadherin, $\mathrm{N}$-cadherin and vimentin. (B) Western blot assays were used to detect the protein expression levels of E-cadherin, $\mathrm{N}$-cadherin and vimentin after treatment with THP-1-CM and/or shikonin for $24 \mathrm{~h}$. (C) Real-time PCR was used to detect the mRNA levels of E-cadherin, N-cadherin and vimentin after treatment with THP-1-CM and/or shikonin for $24 \mathrm{~h}$. $\beta$-actin was used as an internal control. Data are shown as the mean \pm SD of three independent experiments. ${ }^{*} \mathrm{P}<0.05,{ }^{* *} \mathrm{P}<0.01$, compared with the THP-1-CM-treated group. THP-1-CM, THP-1 cell conditioned medium. 
A

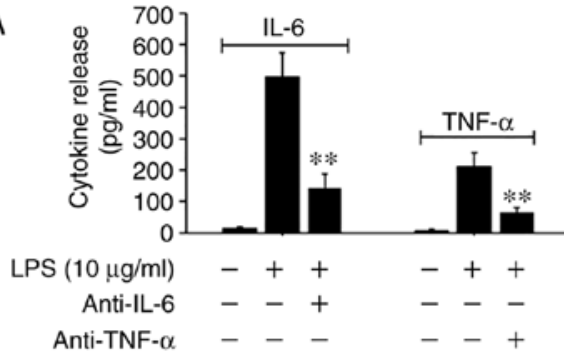

B

A549

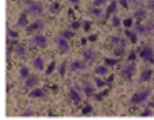

H1299

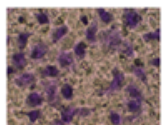

THP-1-CM

Anti-IL-6 Anti-TNF- $\alpha$

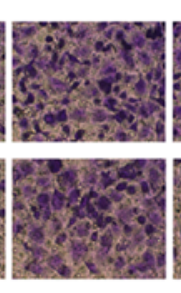

$+$

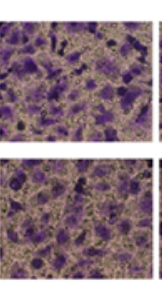

$+$
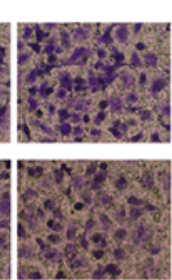

$-$
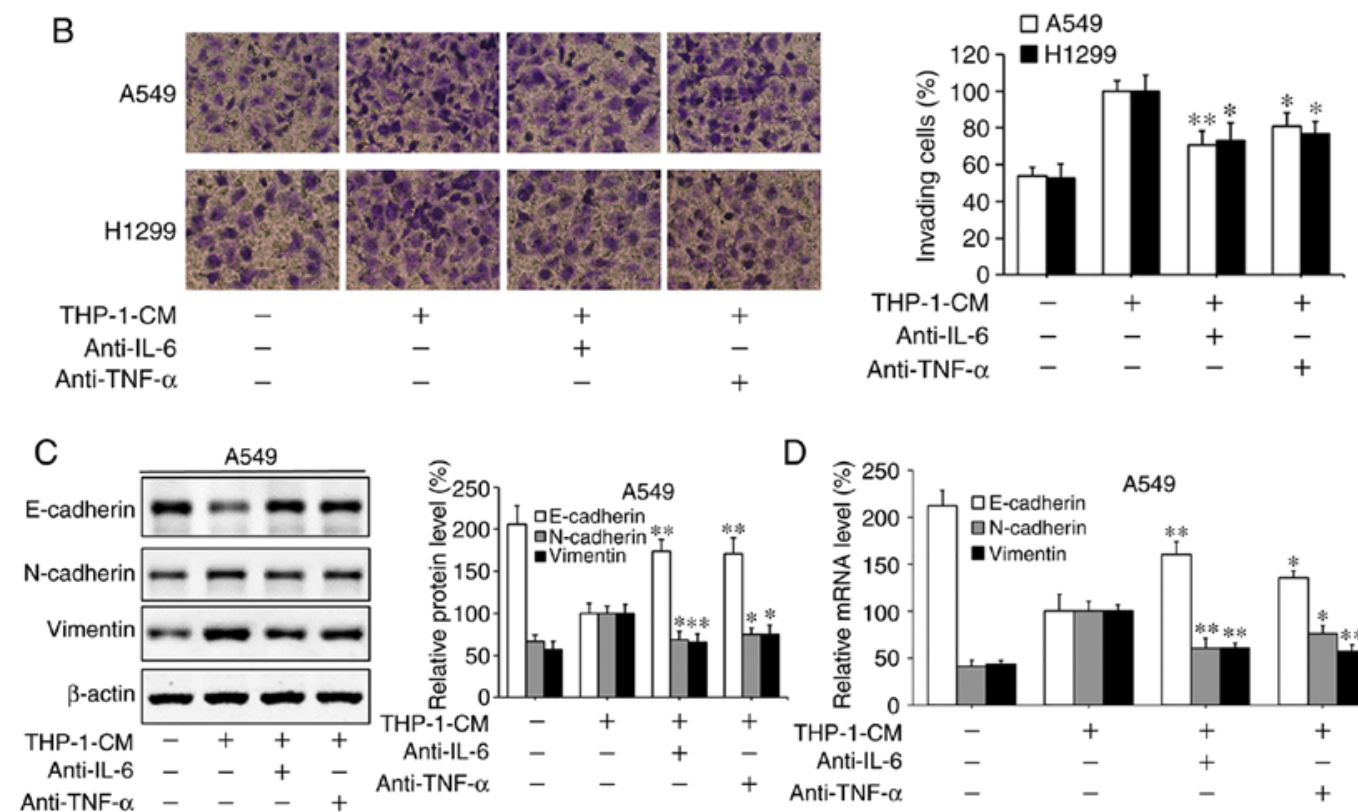

D
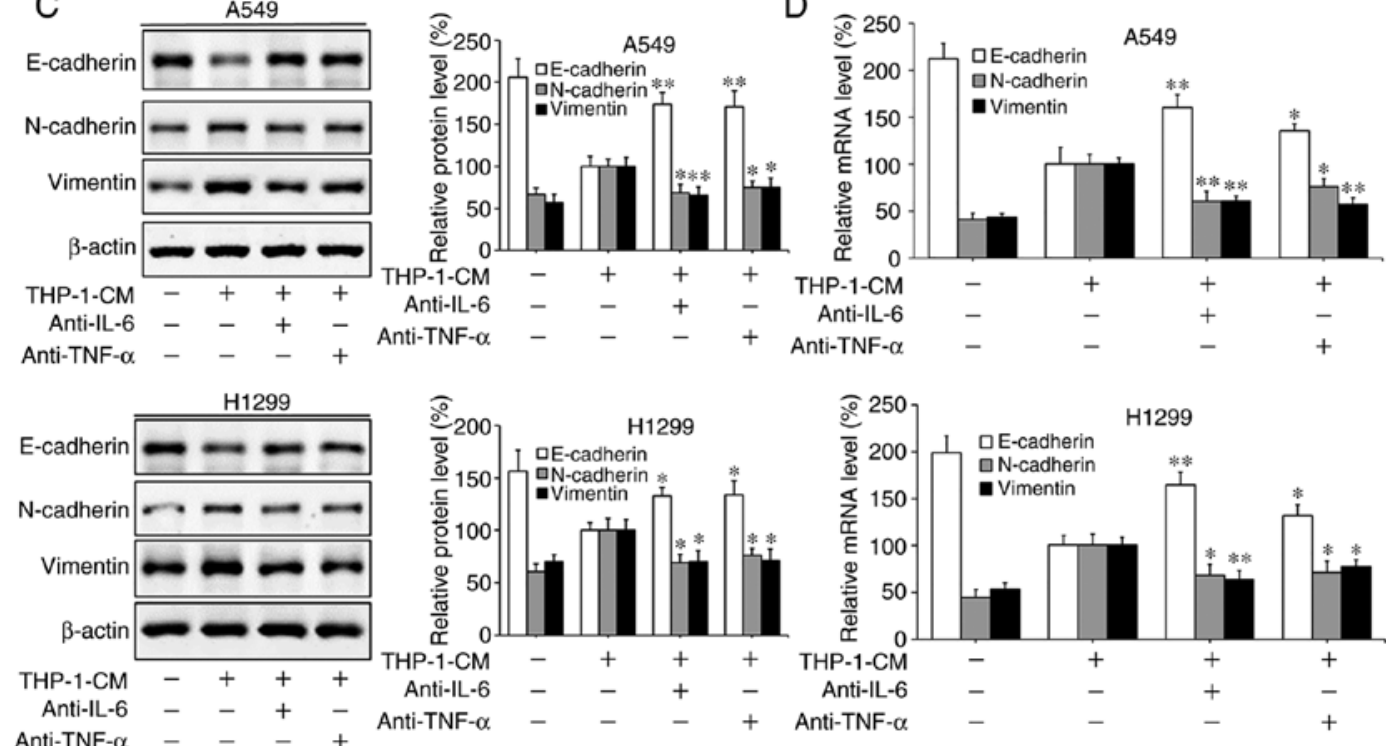

Figure 3. Effect of IL-6 and TNF- $\alpha$ on the migration and epithelial-mesenchymal transition (EMT) of A549 and H1299 cells. (A) IL-6 and TNF- $\alpha$ secreted into the culture supernatants were measured using ELISA kits after treatment with LPS and anti-IL- 6 or anti-TNF- $\alpha$ antibodies for $24 \mathrm{~h}$ in THP-1-CM. ${ }^{* *} \mathrm{P}<0.01$, compared with the corresponding LPS-treated alone group. (B) Effects of 24-h neutralization with anti-IL-6 or anti-TNF- $\alpha$ antibody on the migration of A549 and H1299 cells, as determined by the Transwell chamber migration assay. (C) The protein levels of E-cadherin, N-cadherin and vimentin were detected by western blotting with specific antibodies. Protein expression levels were semi-quantified by densitometry analysis. (D) The relative mRNA levels of E-cadherin, $\mathrm{N}$-cadherin and vimentin were detected by real-time PCR. $\beta$-actin was used as an internal control. Data are shown as the mean \pm SD of three independent experiments. ${ }^{*} \mathrm{P}<0.05,{ }^{* *} \mathrm{P}<0.01$, compared with the THP-1-CM-treated group. THP-1-CM, THP-1 cell conditioned medium; LPS, lipopolysaccharide; IL-6, interleukin-6; TNF- $\alpha$, tumor necrosis factor- $\alpha$.

IL-6 and TNF- $\alpha$ in THP-1-CM trigger EMT in human lung adenocarcinoma cells. Based on previous findings that IL- 6 and TNF- $\alpha$ can promote EMT and migration in tumor cells $(5,6,34)$, we investigated the roles of IL- 6 and TNF- $\alpha$ in THP-1-CM-induced EMT and migration. As shown in Fig. 3A, the levels of IL-6 and TNF- $\alpha$ markedly increased in the supernatants from the LPS-challenged THP-1 cells, with their detectable levels significantly reduced upon the addition of the respective neutralizing antibodies. Moreover, the migration of A549 and H1299 cells induced by THP-1-CM was significantly attenuated by the neutralizing anti-IL-6 antibody (Fig. 3B). The changes in the protein and mRNA expression levels of EMT markers induced by THP-1-CM were also reversed upon the addition of the anti-IL-6 antibody (Fig. 3C and D). Similar phenomena were also observed after the addition of the anti-TNF- $\alpha$ antibody. These results indicate that IL- 6 and TNF- $\alpha$ play important roles in the EMT of lung adenocarcinoma cells.

Shikonin suppresses IL-6-induced migration and EMT in human lung adenocarcinoma cells. To gain further insights of the mechanisms underlying the inhibitory effects of shikonin on EMT induced by THP-1-CM, both cell lines were treated with IL-6 and TNF- $\alpha$ as described previously (5). After cultured in IL-6-supplemented media $(50 \mathrm{ng} / \mathrm{ml})$, both cell lines displayed enhanced migration ability (Fig. 4A), which 

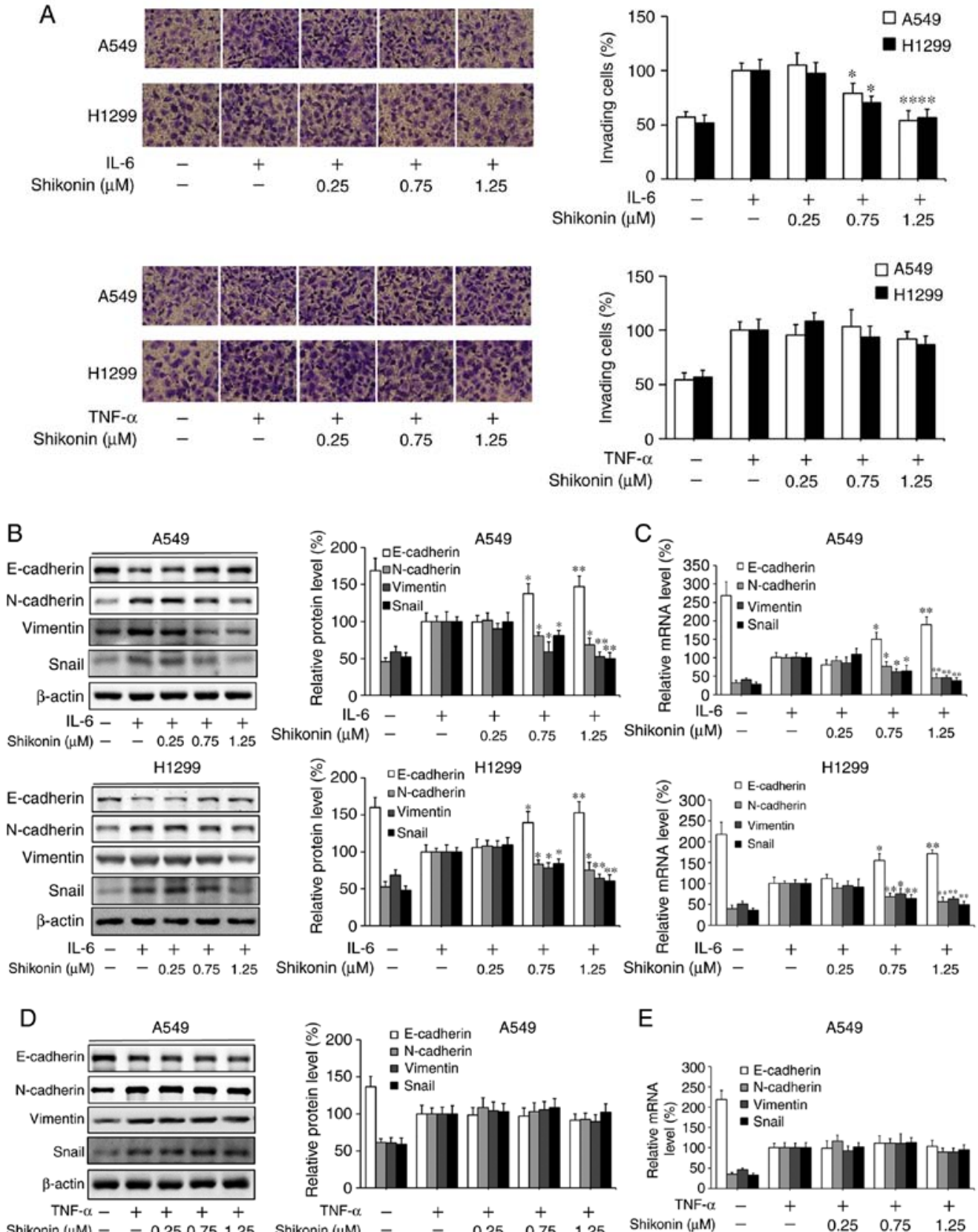

$\mathrm{E}$
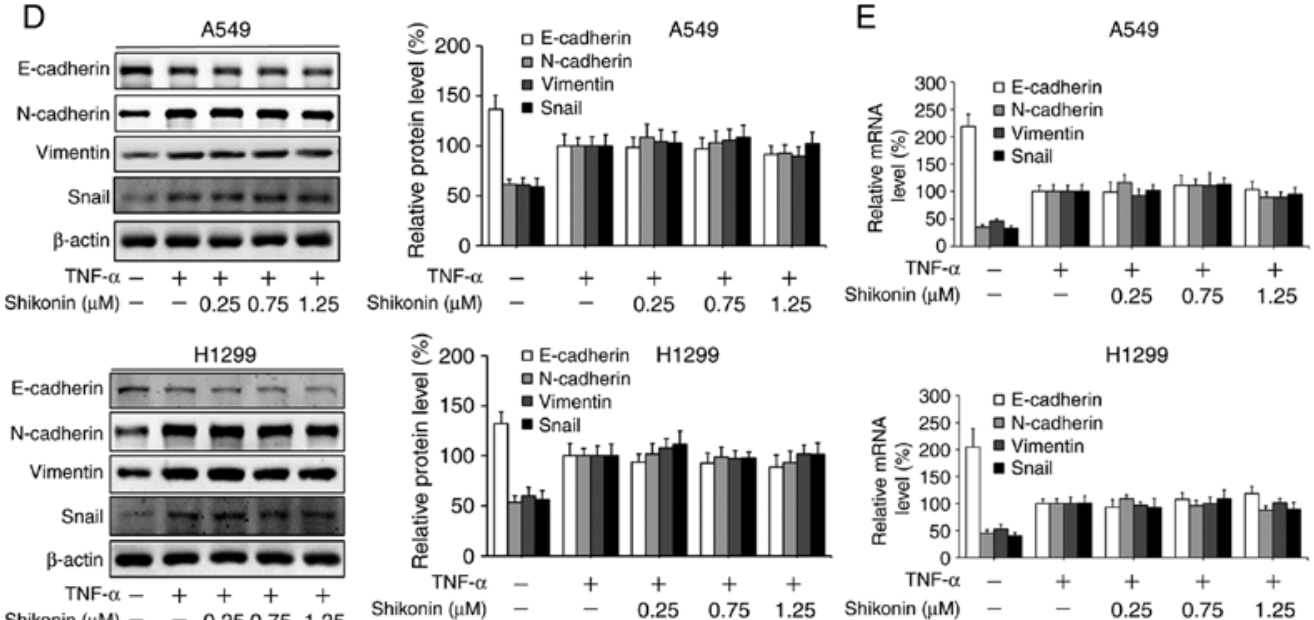

Figure 4. Effects of shikonin on the migration and epithelial-mesenchymal transition (EMT) of A549 and H1299 cells induced by IL-6 and TNF- $\alpha$. (A) Transwell chamber migration assay. After treatment with shikonin and IL-6 (50 ng/ml) or TNF- $\alpha(20 \mathrm{ng} / \mathrm{ml})$ for $24 \mathrm{~h}$, A549 and H1299 cell suspensions were subjected to a Transwell chamber migration assay. (B) Western blot and (C) real-time PCR assays were used to detect the protein and mRNA levels of E-cadherin, $\mathrm{N}$-cadherin, vimentin and Snail in A549 and H1299 cells after treatment with shikonin and IL-6 (50 ng/ml) for 24 h. (D) Western blot and (E) real-time PCR assays were used to detect the protein and mRNA levels of E-cadherin, N-cadherin, vimentin and Snail in A549 and H1299 cells after treatment with shikonin and TNF- $\alpha(20 \mathrm{ng} / \mathrm{ml})$ for $24 \mathrm{~h}$. $\beta$-actin was used as an internal control. Data are shown as the mean $\pm \mathrm{SD}$ of three independent experiments. ${ }^{*} \mathrm{P}<0.05,{ }^{* *} \mathrm{P}<0.01$, compared with the IL-6- or TNF- $\alpha$-treated group. IL-6, interleukin-6; TNF- $\alpha$, tumor necrosis factor $\alpha$.

could be blocked by shikonin administration. Furthermore, the results of western blot analysis and real-time PCR assay revealed that shikonin restored the downregulation of
E-cadherin, while attenuated the upregulation of $\mathrm{N}$-cadherin, vimentin and Snail induced by IL-6 (Fig. 4B and C). However, shikonin did not significantly inhibit the migration 
A
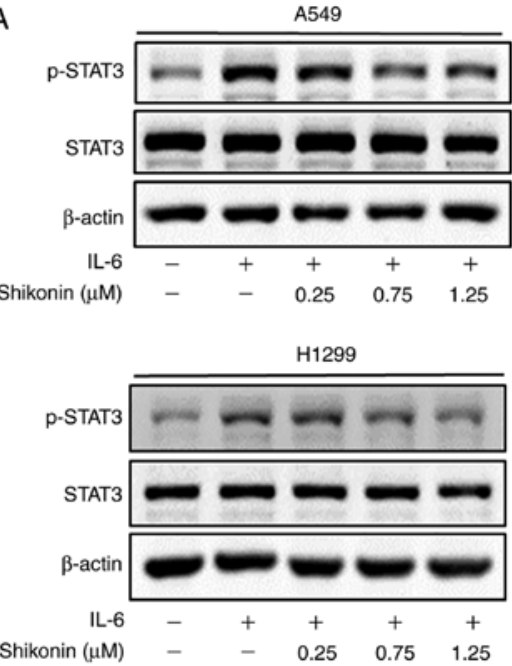

B
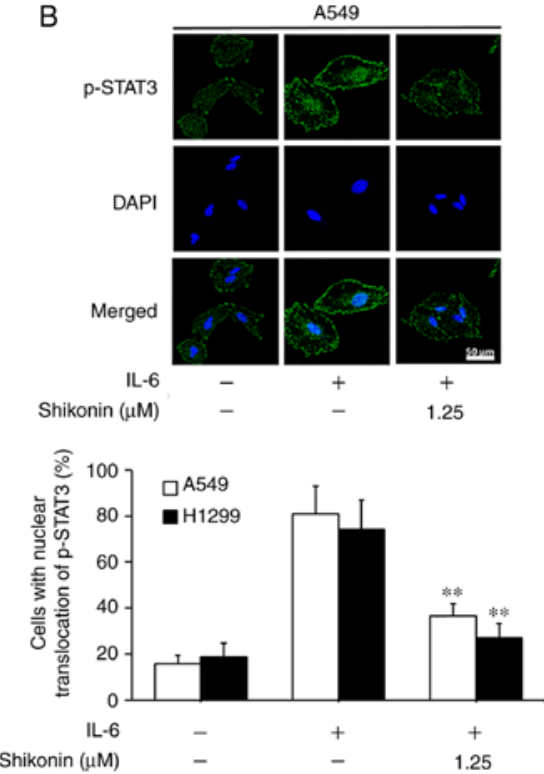

D
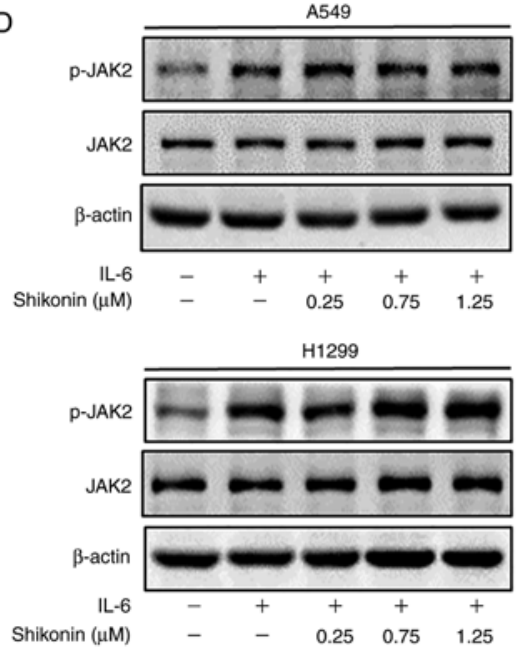
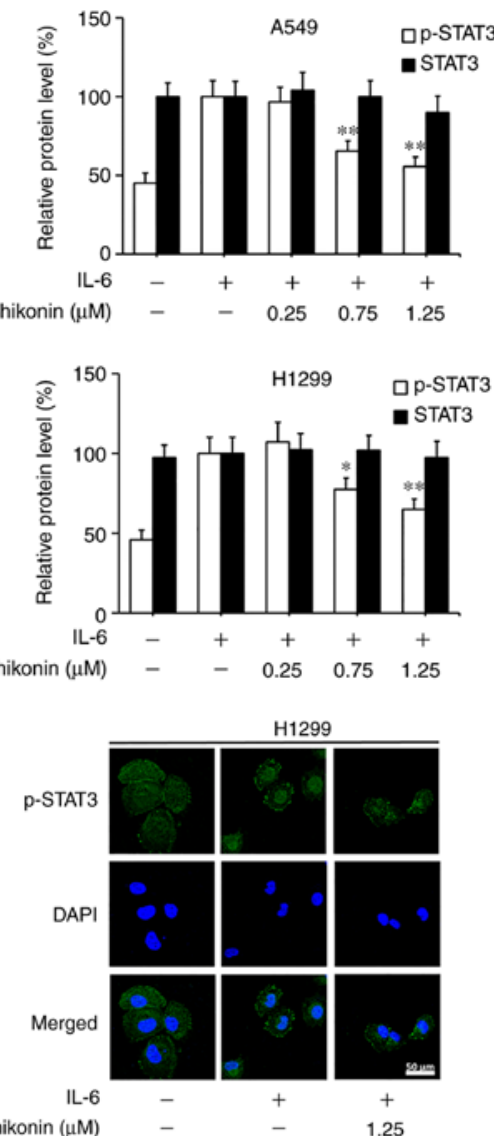

C
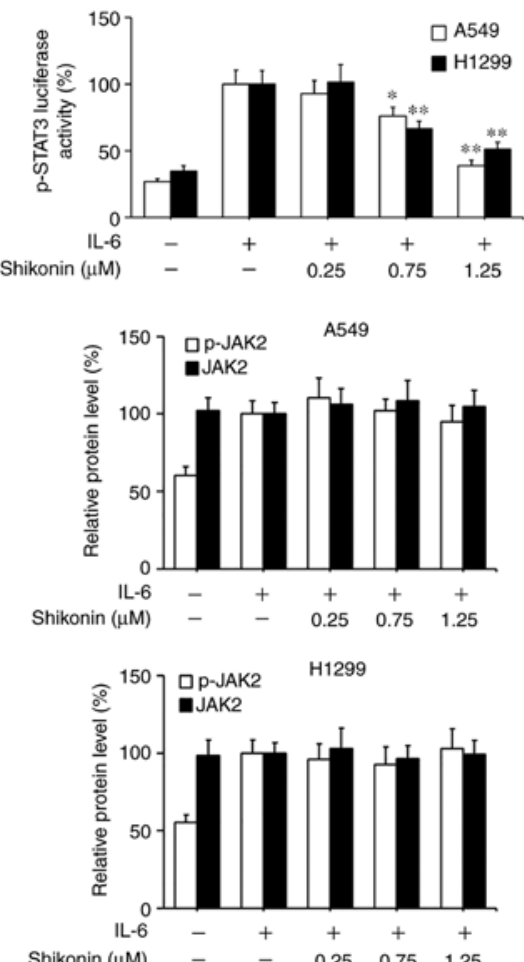

Figure 5. Effects of shikonin on IL-6-induced STAT3 activation in A549 and H1299 cells. (A) Expression levels of phosphorylated (p)-STAT3 and STAT3 were detected by western blotting after treatment with shikonin and IL-6 $(50 \mathrm{ng} / \mathrm{ml})$ for $24 \mathrm{~h}$. Protein expression levels were semi-quantified by densitometric analysis. ${ }^{*} \mathrm{P}<0.05,{ }^{\text {*** }} \mathrm{P}<0.01$, compared with the IL-6-treated group. (B) Immunofluorescence assays were performed to determine the effects of shikonin on the nuclear translocation of p-STAT3 after treatment with shikonin $(1.25 \mu \mathrm{M})$ and IL-6 $(50 \mathrm{ng} / \mathrm{ml})$ for $24 \mathrm{~h}$. Upper panels, representative immunofluorescence images of p-STAT3 staining (magnification $\mathrm{x} 800$ ). Lower panel, the percentage of cells with p-STAT3 nuclear translocation. ${ }^{* *} \mathrm{P}<0.01$, compared with the IL-6-treated group. (C) Transactivation activities of p-STAT3 in A549 and H1299 cells co-transfected with pSTAT3-TA-luc and pRL-TK Renilla, and treated with shikonin and IL-6 (50 ng/ml) for $24 \mathrm{~h}$. Luciferase activity was detected by the Dual Luciferase Reporter kit and normalized against the values for the corresponding pRL-TK Renilla activity. ${ }^{*} \mathrm{P}<0.05$, ${ }^{* *} \mathrm{P}<0.01$, compared with the IL-6-treated group. (D) The protein expression levels of p-JAK2 and JAK2 were detected by western blotting after treatment with shikonin and IL-6 $(50 \mathrm{ng} / \mathrm{ml})$ for $24 \mathrm{~h}$. $\beta$-actin was used as an internal control. Protein expression levels were semi-quantified by densitometry analysis. 

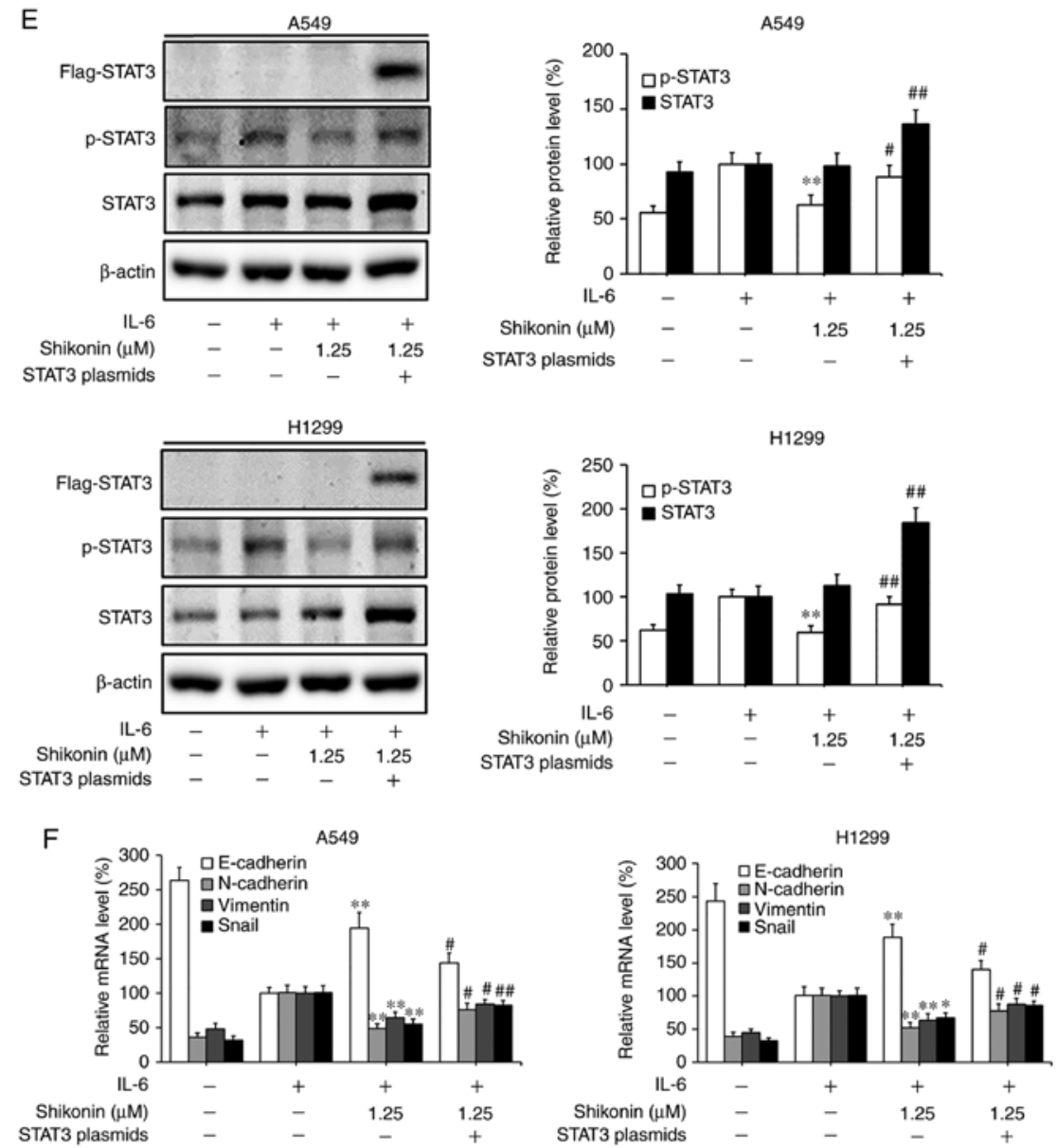

Figure 5. Continued. (E and F) After transfection with STAT3 plasmids for $24 \mathrm{~h}$, the cells were treated with shikonin $(1.25 \mu \mathrm{M})$ and IL-6 (50 ng/ml) for another $24 \mathrm{~h}$. (E) Expression levels of p-STAT3, Flag-STAT3, and STAT3 proteins were detected by western blotting. (F) mRNA expression levels of epithelial-mesenchymal transition (EMT)-related genes (E-cadherin, N-cadherin, vimentin and Snail) were detected by real-time PCR. Data are shown as the mean \pm SD of three independent experiments. ${ }^{*} \mathrm{P}<0.05,{ }^{* *} \mathrm{P}<0.01$, compared with the IL-6-treated group; ${ }^{*} \mathrm{P}<0.05,{ }^{\# \prime} \mathrm{P}<0.01$, compared with the combined shikonin (1.25 $\left.\mu \mathrm{M}\right)$ and IL-6 (50 ng/ml) treatment group. IL-6, interleukin-6; STAT3, signal transducer and activator of transcription 3; JAK, Janus kinase.

and EMT of A549 and H1299 cells induced by $20 \mathrm{ng} / \mathrm{ml}$ TNF- $\alpha$ (Fig. 4D and E), suggesting that only IL-6 and its downstream effectors are responsible for the anti-metastatic effects of shikonin.

Shikonin inhibits IL-6-induced STAT3 activation in human lung adenocarcinoma cells. STAT3 is a downstream factor of IL-6, which hyperactivates in the majority of human cancers and is closely associated with poor prognosis (35). IL-6 can promote the EMT, migration and invasion activities of tumor cells in an inflammatory microenvironment via STAT3 activation and phosphorylation $(3,6)$. As a consequence, the effects of shikonin on IL-6-mediated STAT3 phosphorylation in A549 and H1299 cells were analyzed by western blotting. As shown in Fig. 5A, the addition of IL-6 markedly increased the level of p-STAT3 (Y705), while shikonin treatment significantly suppressed the overexpression of p-STAT3 induced by IL-6 in a dose-dependent manner without affecting the total STAT3 level. In addition, the results of immunofluorescence and luciferase reporter gene assays indicated that shikonin could prevent the nuclear translocation of p-STAT3 (Fig. 5B) and inhibit its transactivation activity in both cell lines (Fig. 5C). However, there were no significant inhibitory effects of shikonin on both p-JAK2 or JAK2 levels (Fig. 5D). To further verify whether shikonin can exert its inhibitory effects through the IL-6/STAT3 pathway, pcDNA3.1-Flag-STAT3 was used to overexpress STAT3 in A549 and H1299 cells. As shown in Fig. 5E and F, after STAT3 overexpression, the inhibitory effects of shikonin on STAT3 phosphorylation and EMT induced by IL- 6 were alleviated. Therefore, these results indicate that shikonin can reverse IL-6-induced EMT by inhibiting the activation of STAT3 in human lung adenocarcinoma cells.

Shikonin inhibits the metastasis of lung tumors in vivo. An artificial lung metastasis model was used to study the anti-metastatic effects of shikonin in vivo $(5,6)$. In the present study, shikonin treatment at 2.5 and $5 \mathrm{mg} / \mathrm{kg}$ significantly inhibited lung metastasis, as shown in the image of lung tissue and H\&E staining assays (Fig. 6A). The number of lung metastatic nodules was $102 \pm 20$ in the vehicle control group, while only $45 \pm 11$ and $24 \pm 7$ nodules were found in the groups treated with 2.5 and $5 \mathrm{mg} / \mathrm{kg}$ shikonin, respectively (Fig. 6B). These results suggest that shikonin can effectively suppress the metastasis of lung adenocarcinoma in vivo.

The effects of shikonin on the growth of lung tumors were studied using a nude mice xenograft model. As shown in Fig. $6 \mathrm{C}$ and $\mathrm{D}$, the volumes and weights of tumor xenografts 
A

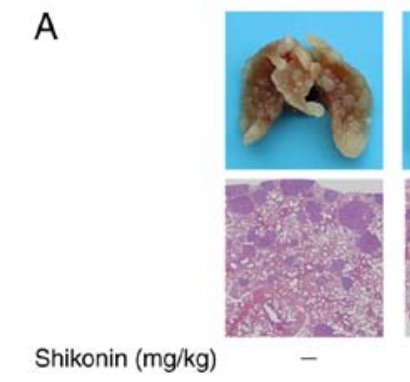

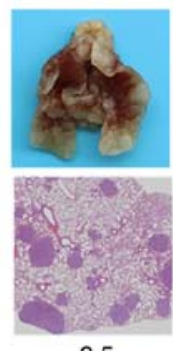

2.5

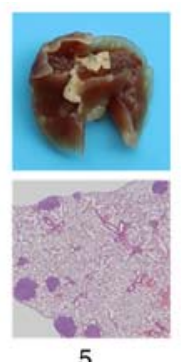

5
B

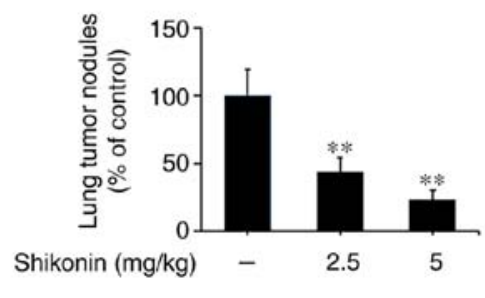

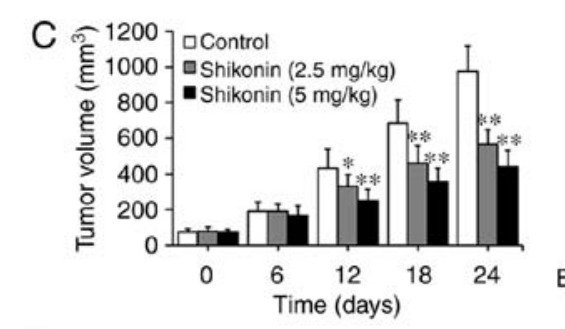

E
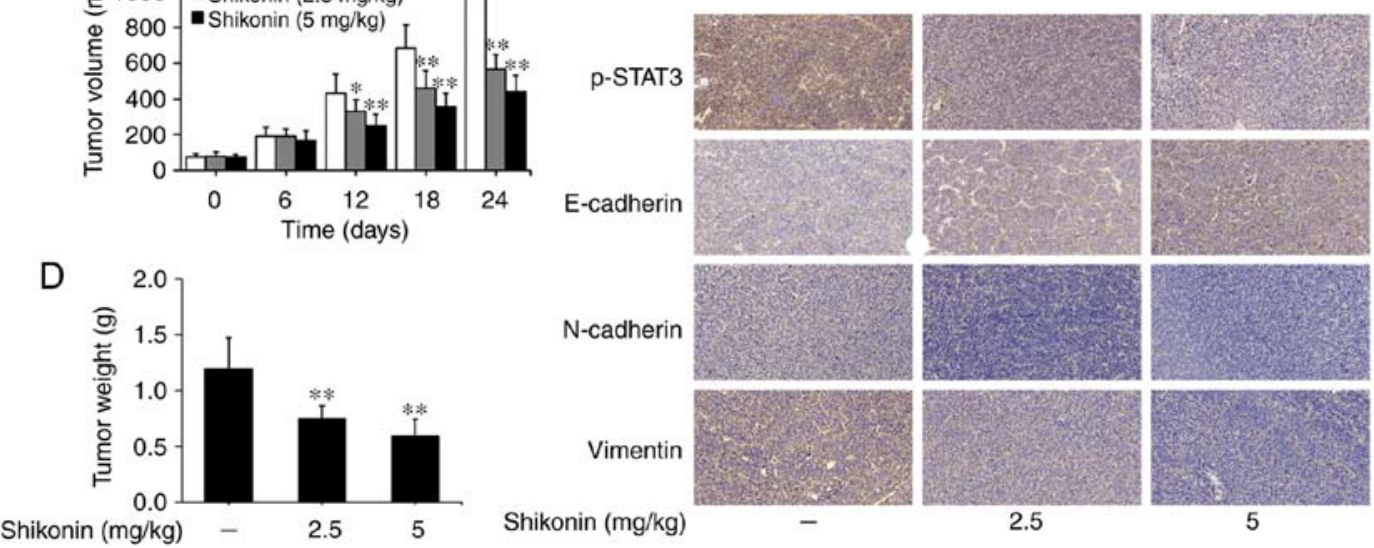

5

Figure 6. Effects of shikonin on tumor metastasis and growth in vivo. (A) Representative images of lung tissue and hematoxylin and eosin (H\&E)-stained metastatic lung nodules. (B) Quantification of the number of metastatic nodules on lung surface. (C) Tumor volume and (D) weight of the control and shikonin treatment groups. (E) Representative immunohistochemistry images of p-STAT3, E-cadherin, N-cadherin and vimentin expression in the tumor tissues derived from the A549 xenografts. Data are presented as mean $\pm \mathrm{SD},{ }^{*} \mathrm{P}<0.05,{ }^{* *} \mathrm{P}<0.01$, compared with the control group. p-STAT3, phosphorylated signal transducer and activator of transcription 3.

were significantly reduced in the shikonin treatment groups compared to the vehicle control group. Furthermore, through immunohistochemical staining, it was found that the expression levels of p-STAT3, N-cadherin and vimentin were decreased, while E-cadherin expression was increased in tumor xenografts treated with shikonin (Fig. 6E). Taken together, these data suggest that shikonin can prevent tumor metastasis and EMT by inhibiting STAT3 activation in vivo.

IL-6 expression is upregulated in human lung adenocarcinoma tissues. As presented in Table I, the expression level of IL-6 in tumor tissues was significantly associated with tumor-node-metastasis (TNM) stage and lymph node metastasis, but not with sex and age. Then, we further compared the expression levels of IL-6 levels in lung adenocarcinoma tissues and matched normal lung tissues. As shown in Fig. 7A, the positive staining of IL- 6 was mainly observed in the cytoplasm of tumor cells and inflammatory cells (e.g. macrophages), and the levels of IL-6 were significantly elevated in lung adenocarcinoma tissues compared to these level in the normal lung tissues (Fig. 7B). Furthermore, the distribution of tumor-associated macrophages (TAMs) was assessed by the well-characterized marker CD68. Although there was no significant difference in CD68 levels between lung adenocarcinoma tissues and normal lung tissues (data not shown), the level of CD68 was significantly positively correlated with IL-6 expression in lung adenocarcinoma tissues $(\mathrm{R}=0.399, \mathrm{P}<0.05$; Fig. 7C and D).

\section{Discussion}

As a physiological process mediated by the innate immune system, inflammation can protect the body against pathogenic infections, environmental insults and injuries. However, recent studies have demonstrated that inflammation and tumors are closely associated (36). It has been shown that the inflammatory microenvironment, which is an essential component of the tumor microenvironment, plays a key role during tumor promotion and metastasis (37). Inflammatory cells, such as tumor-associated macrophages (TAMs), can induce tumor angiogenesis, extracellular matrix breakdown and tissue remodeling, thus aggravating tumor metastasis (38). In addition, epithelial-mesenchymal transition (EMT) induction in the inflammatory microenvironment can promote the metastatic ability of tumor cells (39). EMT in tumor cells is a process of the loss of cell-cell adhesion ability as well as acquiring migratory and invasive properties. During this process, the levels of epithelial markers, such as E-cadherin, are decreased, while those of mesenchymal markers, such as $\mathrm{N}$-cadherin and vimentin, are increased (40). In the present study, we used LPS-stimulated THP-1-CM to mimic the inflammatory microenvironment in vitro according to previously reported methods $(6,41,42)$. It was found that shikonin could reverse EMT and inhibit the migration and invasion of human lung adenocarcinoma cells within the in vitro inflammatory microenvironment. Although our experiments could not completely mimic the event of tumors cells being persistently exposed to the inflammatory microenvironment in vivo, our 
Table I. Association between IL-6 expression and clinicopathological features of the patients with lung adenocarcinoma (N=128).

IL-6 expression

Clinical parameters

$\mathrm{Low}^{\mathrm{a}}, \mathrm{n}$

$\mathrm{High}^{\mathrm{b}}, \mathrm{n}$

P-value

Sex

Male

24

30

Female

TNM stage

II

Lymph node metastasis

$\mathrm{N}_{0}$

$\mathrm{N}_{1} / \mathrm{N}_{2} / \mathrm{N}_{3}$

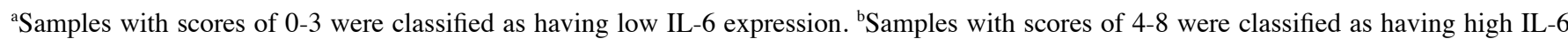
expression. ${ }^{c} \mathrm{P}<0.05$ and ${ }^{\mathrm{d}} \mathrm{P}<0.01$ represent a significant difference. IL-6, interleukin 6.

A

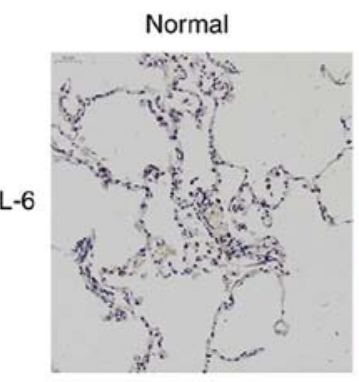

C

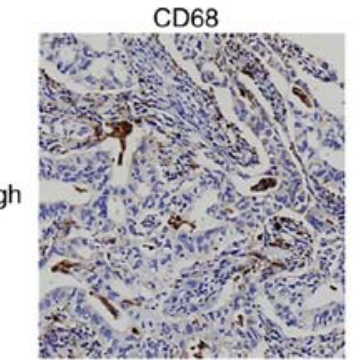

CD68

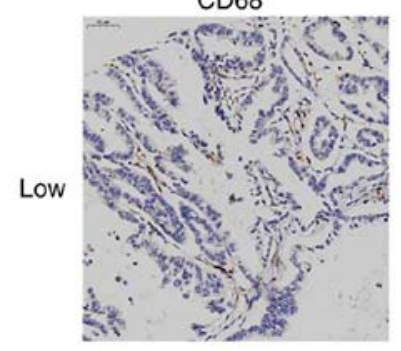

Tumor

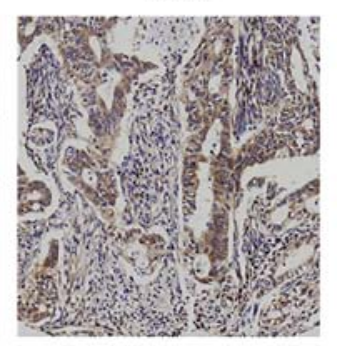

IL-6

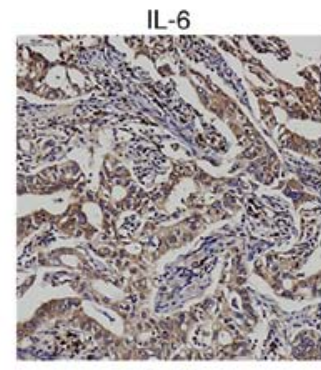

IL-6

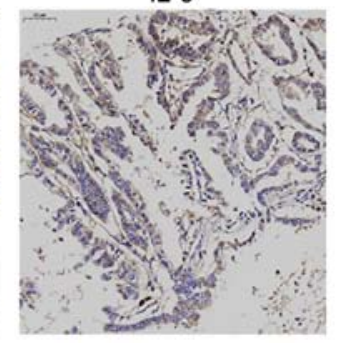

23

59

3

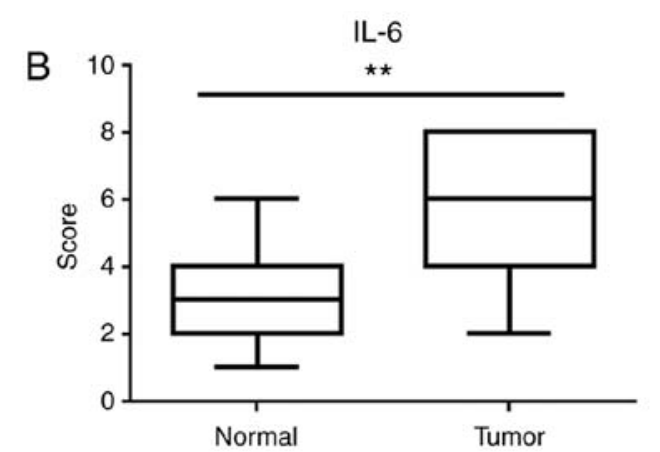

D

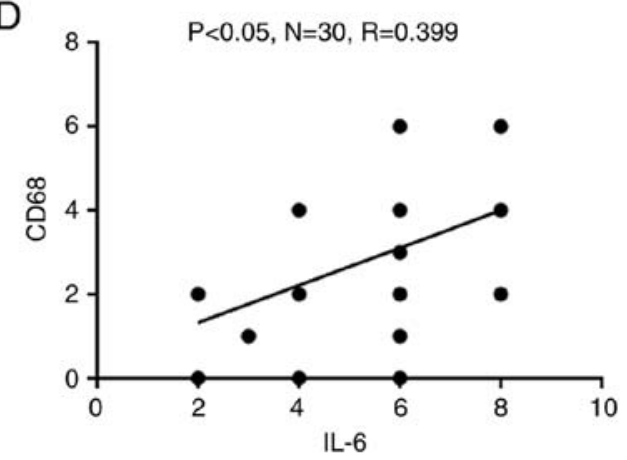

Figure 7. IL-6 levels are higher in human lung adenocarcinoma tissues and corresponding TAM infiltration. (A) Representative immunohistochemistry images of IL-6 in lung adenocarcinoma tissues and matched normal lung tissues (magnification x200). (B) Statistical analysis of the immunohistochemistry scores for the expression of IL- 6 in 30 pairs of lung adenocarcinoma tissues and matched normal lung tissues, ${ }^{* *} \mathrm{P}<0.01$, compared with the matched normal lung tissue group. (C) Representative immunohistochemistry images with high and low levels of IL-6 and CD68 (magnification x200). (D) Scatter chart showing a positive correlation between the expression levels of IL-6 and CD68 in 30 pairs of lung adenocarcinoma tissues. IL-6, interleukin-6; TAM, tumor-associated macrophage. 
results at least indicated that shikonin could inhibit EMT in lung adenocarcinoma cells exposed to soluble inflammatory factors secreted by LPS-stimulated THP-1 cells. Altogether, these findings suggest that shikonin possesses a potent anti-metastatic effect within an inflammatory microenvironment.

Inflammatory cell infiltrates can secrete various inflammatory cytokines into tumor cells to activate the major inflammatory signaling pathways and facilitate tumor progression and metastasis $(43,44)$. IL-6, an important inflammatory cytokine that regulates systemic and regional inflammation, can promote the pathogenesis of tumors by enhancing tumor metastasis, recruiting leukocytes and promoting angiogenesis (35). In the present study, the addition of the anti-IL-6 antibody into THP-1-CM was able to inhibit the migration and EMT of lung adenocarcinoma cells, suggesting that IL-6 directly promotes EMT in an inflammatory microenvironment. In addition, shikonin markedly reversed EMT and inhibited IL-6-induced migration of lung adenocarcinoma cells. These findings suggest that shikonin can exert anti-metastatic effects in an inflammatory microenvironment via the IL-6-mediated signaling pathway.

As the downstream factor of IL-6, STAT3 is hyperactivated in various human cancers, displays tumor-promoting properties, and is associated with poor clinical prognosis (35). Experimental studies have found that activated STAT3 can regulate the expression levels of EMT markers through Snail and Slug transcription factors, thus aggravating EMT and tumor metastasis $(17,45)$. In the IL-6/STAT3 signaling pathway, the binding of cytokine IL-6 to the IL-6R complex, which consists of IL-6R and gp130, can activate the JAK2 pathway. The activation of JAK2 can phosphorylate STAT3 at Y705. p-STAT3 monomers can form dimers and subsequently translocate into the nucleus to initiate the transcription of its target genes (6). In this study, we found that shikonin could inhibit IL-6-induced phosphorylation of STAT3, prevent p-STAT3 from translocating into the nucleus, and suppress the transactivation activity of p-STAT3. Furthermore, the overexpression of STAT3 attenuated shikonin-inhibited STAT3 phosphorylation and EMT in lung adenocarcinoma cells upon exposure to IL-6. These findings revealed that shikonin can inhibit IL-6-induced EMT by inhibiting the activation of STAT3 in lung adenocarcinoma cells. However, shikonin did not inhibit IL-6-induced phosphorylation of JAK2 under our experimental conditions. Based on previous studies, we speculate that shikonin may inhibit JAK2-mediated activation of STAT3 by binding directly to the STAT3 SH2 domain $(46,47)$. However, more studies are needed to identify the exact mechanism of action.

Moreover, artificial lung metastasis and xenograft models were used to determine the effects of shikonin on human lung adenocarcinoma metastasis and growth in vivo. It was found that shikonin treatment markedly suppressed the metastasis and growth of lung adenocarcinoma. In addition, it was also noted that shikonin inhibited STAT3 activation and EMT in a lung adenocarcinoma xenograft. Furthermore, the results of tissue microarrays indicated that the expression level of IL-6 was higher in human lung adenocarcinoma tissues than that noted in normal lung tissues. More importantly, the expression levels of IL-6 in human lung adenocarcinoma tissues were significantly associated with TNM stage and lymph node metastasis. Taken together, our results demonstrate that shikonin has great potential for the suppression of lung adenocarcinoma metastasis by inhibiting STAT3 activation in vivo.

Notably, we speculate that other cytokines such as IL-1 $\beta$ or IL- 8 may also be expressed in THP-1-CM $(48,49)$. Actually, according to the general principle, most cytokines that can be secreted by THP-1 cells after LPS stimulation should be expressed in THP-1-CM. Whether shikonin has effects on the other cytokines and their downstream pathways still requires further investigations.

In conclusion, this study reports, for the first time, that shikonin suppresses the EMT, migration and invasion activities of human lung adenocarcinoma cells within the inflammatory microenvironments involving the IL-6/STAT3 signaling pathway. These findings provide additional insights into the antitumor activities of shikonin, which may have valuable implications for treating inflammation-related tumor metastasis.

\section{Acknowledgements}

The authors would like to thank the staff of Rui Zhang's laboratory for offering their assistance.

\section{Funding}

The present study was supported by grants from the National Natural Science Foundation of China (grant nos. 8187102538, 81872421, 81172222, 81572504 and 81502405); the Natural Science Foundation of Shaanxi Province (grant nos. 2019JQ889, 2017JM8086 and 2016JZ028); the State Key Laboratory of Cancer Biology of China (grant nos. CBSKL201710 and CBSKL2017Z09); and the Foundation of Xi'an Medical University (grant no. 2018DOC02).

\section{Availability of data and materials}

The datasets generated and analyzed during the present study are available from the corresponding author on reasonable request.

\section{Authors' contributions}

TP and FZ contributed equally to this study. TP, XR and ZL conceived and designed the experiments. TP and FZ carried out the experiments in the study. FL and XG assisted in performing the experiments. FZ analyzed the data and constructed the figures. TP and FZ wrote the manuscript. XL revised the manuscript. All authors read and approved the manuscript and agree to be accountable for all aspects of the research in ensuring that the accuracy or integrity of any part of the work are appropriately investigated and resolved.

\section{Ethics approval and consent to participate}

The experiments involving human subjects were approved by the Institutional Review Board of Xi Jing Hospital of The Fourth Military Medical University (Xi'an, Shaanxi, China), and in accordance with the relevant guidelines and regulations. All animal studies were approved by the Animal Ethics Committee of The Fourth Military Medical University (Xi'an, Shaanxi, China). 


\section{Patient consent for publication}

Not applicable.

\section{Competing interests}

The authors declare that they have no competing interests.

\section{References}

1. Yang D, Cao X, Wang F, Jiang, Feng D, Guo H, Du L, Jin Y, Chen Y, Yin X and Li C: LFG-500, a novel synthetic flavonoid, suppresses epithelial-mesenchymal transition in human lung adenocarcinoma cells by inhibiting NLRP3 in inflammatory microenvironment. Cancer Lett 400: 137-148, 2017.

2. Collins LG, Haines C, Perkel R and Enck RE: Lung cancer: Diagnosis and management. Am Fam Physician 75: 56-63, 2007.

3. Zhao Z, Cheng X, Wang Y, Han R, Li L, Xiang T, He L, Long H, Zhu B and He Y: Metformin inhibits the IL-6-induced epithelial-mesenchymal transition and lung adenocarcinoma growth and metastasis. PLoS One 9: e95884, 2014.

4. Li J, Deng Z, Wang Z, Wang D, Zhang L, Su Q, Lai Y, Li B, Luo Z, Chen X, et al: Zipper-Interacting protein kinase promotes epithelial-mesenchymal transition, invasion and metastasis through AKT and NF-kB signaling and is associated with metastasis and poor prognosis in gastric cancer patients. Oncotarget 6 : 8323-8338, 2015

5. Song J, Feng L, Zhong R, Xia Z, Zhang L, Cui L, Yan H, Jia X and Zhang Z: Icariside II inhibits the EMT of NSCLC cells in inflammatory microenvironment via down-regulation of Akt/NF-kappaB signaling pathway. Mol Carcinog 56: 36-48, 2017.

6. Zhao Y, Yao J, Wu XP, Zhao L, Zhou YX, Zhang Y, You QD, Guo QL and Lu N: Wogonin suppresses human alveolar adenocarcinoma cell A549 migration in inflammatory microenvironment by modulating the IL-6/STAT3 signaling pathway. Mol Carcinog 54: E81-E93, 2015.

7. MicalizziDS,Farabaugh SMandFordHL:Epithelial-mesenchymal transition in cancer: parallels between normal development and tumor progression. J Mammary Gland Biol Neoplasia 15: 117-134, 2010.

8. Mantovani A, Allavena P, Sica A and Balkwill F: Cancer-Related inflammation. Nature 454: 436-444, 2008.

9. DeNardo DG, Andreu P and Coussens LM: Interactions between lymphocytes and myeloid cells regulate pro- versus anti-tumor immunity. Cancer Metastasis Rev 29: 309-316, 2010.

10. Ostrand-Rosenberg S: Immune surveillance: A balance between pro- and anti-tumor immunity. Curr Opin Genet Dev 18: 11-18, 2008 .

11. Park EJ, Lee JH, Yu GY, He G, Ali SR, Holzer RG, Osterreicher $\mathrm{CH}$, Takahashi $\mathrm{H}$ and Karin $\mathrm{M}$ : Dietary and genetic obesity promote liver inflammation and tumorigenesis by enhancing IL-6 and TNF expression. Cell 140: 197-208, 2010

12. Yeh HH, Lai WW, Chen HH, Liu HS and Su WC: Autocrine IL-6-induced Stat3 activation contributes to the pathogenesis of lung adenocarcinoma and malignant pleural effusion. Oncogene 25: 4300-4309, 2006.

13. Lederle W, Depner S, Schnur S, Obermueller E, Catone N, Just A, Fusenig NE and Mueller MM: IL-6 promotes malignant growth of skin SCCs by regulating a network of autocrine and paracrine cytokines. Int J Cancer 128: 2803-2814, 2011.

14. Bromberg J and Wang TC: Inflammation and cancer: IL-6 and STAT3 complete the link. Cancer Cell 15: 79-80, 2009.

15. Heinrich PC, Behrmann I, Haan S, Hermanns HM, Müller-Newen G and Schaper F: Principles of interleukin (IL)-6-type cytokine signalling and its regulation. Biochem J 374: 1-20, 2003.

16. Huang C, Yang G, Jiang T, Zhu G, Li H and Qiu Z: The effects and mechanisms of blockage of STAT3 signaling pathway on IL-6 inducing EMT in human pancreatic cancer cells in vitro. Neoplasma 58: 396-405, 2011.

17. Yadav A, Kumar B, Datta J, Teknos TN and Kumar P: IL-6 promotes head and neck tumor metastasis by inducing epithelial-mesenchymal transition via the JAK-STAT3-SNAIL signaling pathway. Mol Cancer Res 9: 1658-1667, 2011.
18. Zhou G, Yang Z, Wang X, Tao R and Zhou Y: TRAIL Enhances shikonin induced apoptosis through ros/jnk signaling in cholangiocarcinoma cells. Cell Physiol Biochem 42: 1073-1086, 2017.

19. Andujar I, Ríos JL, Giner RM and Recio MC: Pharmacological properties of shikonin - A review of literature since 2002. Planta Med 79: 1685-1697, 2013.

20. Chen X, Yang L, Oppenheim JJ and Howard MZ: Cellular pharmacology studies of shikonin derivatives. Phytother Res 16 199-209, 2002

21. Chen J, Xie J, Jiang Z, Wang B, Wang Y and Hu X: Shikonin and its analogs inhibit cancer cell glycolysis by targeting tumor pyruvate kinase-M2. Oncogene 30: 4297-4306, 2011.

22. Yeh YC, Liu TJ and Lai HC: Shikonin induces apoptosis, necrosis, and premature senescence of human a549 lung cancer cells through upregulation of p53 expression. Evid Based Complement Alternat Med 2015: 620383, 2015.

23. Andújar I, Recio MC, Giner RM and Ríos JL: Traditional Chinese medicine remedy to jury: The pharmacological basis for the use of shikonin as an anticancer therapy. Curr Med Chem 20: 2892-2898, 2013.

24. Heinrich EL, Walser TC, Krysan K, Liclican EL, Grant JL, Rodriguez NL and Dubinett SM: The inflammatory tumor microenvironment, epithelial mesenchymal transition and lung carcinogenes. Cancer microenvironment: official journal of the International Cancer Microenv 5: 5-18, 2012

25. Zheng Y, Guo J, Zhou J, Lu J, Chen Q, Zhang C, Qing C, Koeffler HP and Tong Y: FoxM1 transactivates PTTG1 and promotes colorectal cancer cell migration and invasion. BMC Med Genomics 8: 49, 2015.

26. Kuo CL, Lai KC, Ma YS, Weng SW, Lin JP and Chung JG: Gallic acid inhibits migration and invasion of SCC-4 human oral cancer cells through actions of NF-kappaB, ras and matrix metalloproteinase-2 and -9. Oncol Rep 32: 355-361, 2014.

27. Wallmeyer L, Dietert K, Sochorová M, Gruber AD, Kleuser B, Vávrová $\mathrm{K}$ and Hedtrich S: TSLP is a direct trigger for T cell migration in filaggrin-deficient skin equivalents. Sci Rep 7: 774, 2017.

28. Schneider CA, Rasband WS and Eliceiri KW: NIH Image to imagej: 25 years of image analysis. Nat Methods 9: 671-675, 2012.

29. Pan T, Zhang M, Zhang F, Yan G, Ru Y, Wang Q, Zhang Y, Wei X, $\mathrm{Xu} X$, Shen L, et al: NDRG2 overexpression suppresses hepatoma cells survival during metabolic stress through disturbing the activation of fatty acid oxidation. Biochem Biophys Res Commun 5: 860-866, 2017.

30. Livak KJ and Schmittgen TD: Analysis of relative gene expression data using real-time quantitative PCR and the 2(-Delta Delta C(T)) method. Methods 25: 402-408, 2001.

31. Zhao M, Liu Y, Liu R, Qi J, Hou Y, Chang J and Ren L: Upregulation of IL-11, an IL-6 family cytokine, promotes tumor progression and correlates with poor prognosis in non-small cell lung cancer. Cell Physiol Biochem 45: 2213-2224, 2018.

32. Thakur R, Trivedi R, Rastogi N, Singh M and Mishra DP: Inhibition of STAT3, FAK and Src mediated signaling reduces cancer stem cell load, tumorigenic potential and metastasis in breast cancer. Sci Rep 5: 10194, 2015.

33. Wang S, Wu X, Chen Y,Zhang J, Ding J,Zhou Y,He S, Tan Y, Qiang F, Bai J, et al: Prognostic and predictive role of JWA and XRCC1 expressions in gastric cancer. Clin Cancer Res 18: 2987-2996, 2012.

34. Wang $\mathrm{H}$, Wang $\mathrm{HS}$, Zhou BH, Li CL, Zhang $\mathrm{F}$, Wang XF, Zhang G, $\mathrm{Bu}$ XZ, Cai SH and Du J: Epithelial-mesenchymal transition (EMT) induced by TNF-alpha requires AKT/GSK-3beta-mediated stabilization of snail in colorectal cancer. PLoS One 8: e56664, 2013.

35. Johnson DE, O'Keefe RA and Grandis JR: Targeting the IL-6/JAK/STAT3 signalling axis in cancer. Nat Rev Clin oncol 15: 234-248, 2018

36. Diakos CI, Charles KA, McMillan DC and Clarke SJ: Cancer-Related inflammation and treatment effectiveness. Lancet Oncol 15: e493-e503, 2014.

37. Pietila M, Ivaska J and Mani SA: Whom to blame for metastasis, the epithelial-mesenchymal transition or the tumor microenvironment? Cancer Lett 380: 359-368, 2016

38. Bingle L, Brown NJ and Lewis CE: The role of tumour-associated macrophages in tumour progression: Implications for new anticancer therapies. J Pathol 196: 254-265, 2002.

39. Dehai C, Bo P, Qiang T, Lihua S, Fang L, Shi J, Jingyan C, Yan Y, Guangbin $\mathrm{W}$ and Zhenjun Y: Enhanced invasion of lung adenocarcinoma cells after co-culture with THP-1-derived macrophages via the induction of EMT by IL-6. Immunol Lett 160: 1-10, 2014.

40. Xie YG, Yu Y, Hou LK, Wang X, Zhang B and Cao XC: FYN promotes breast cancer progression through epithelial-mesenchymal transition. Oncol Rep 36: 1000-1006, 2016. 
41. Budai MM, Varga A, Milesz S, Tőzsér J and Benkő S: Aloe vera downregulates LPS-induced inflammatory cytokine production and expression of NLRP3 inflammasome in human macrophages. Mol Immunol 56: 471-479, 2013.

42. Wang HW, Wu T, Qi JY, Wang YQ, Luo XP and Ning Q: Salidroside attenuates LPS-stimulated activation of THP-1 cell-derived macrophages through down-regulation of MAPK/NF-kB signaling pathways. J Huazhong Univ Sci Technol Med Sci 33: 463-469, 2013.

43. Candido J and Hagemann T: Cancer-related inflammation. J Clin Immunol 33: S79-S84, 2013.

44. Lesina M, Wörmann SM, Neuhöfer P, Song L and Algül H: Interleukin- 6 in inflammatory and malignant diseases of the pancreas. Semin Immunol 26: 80-87, 2014.

45. Wu YS, Chung I, Wong WF, Masamune A, Sim MS and Looi CY: Paracrine IL-6 signaling mediates the effects of pancreatic stellate cells on epithelial-mesenchymal transition via Stat3/Nrf2 pathway in pancreatic cancer cells. Biochim Biophys Acta Gen Subj 1861: 296-306, 2017.

46. Qiu HY, Zhu X, Luo YL, Lin HY, Tang CY, Qi JL, Pang YJ, Yang RW, Lu GH, Wang XM and Yang Yh: Identification of new shikonin derivatives as antitumor agents targeting stat 3 sh2 domain. Sci Rep 7: 2863, 2017.
47. Qiu HY, Fu JY, Yang MK, Han HW, Wang PF, Zhang YH, Lin HY, Tang CY, Qi JL, Yang RW, et al: Identification of new shikonin derivatives as STAT3 inhibitors. Biochem Pharmacol 146: 74-86, 2017.

48. Kim YI, Choi KH, Kim SR, Goo TW and Park SW: Bombyx mori hemocyte extract has anti-inflammatory effects on human phorbol myristate acetate-differentiated THP1 cells via TLR4-mediated suppression of the NF-kappaB signaling pathway. Mol Med Rep 16: 4001-4007, 2017.

49. Liu X, Yin S, Chen Y, Wu Y, Zheng W, Dong H, Bai Y, Qin Y, Li J, Feng S, and Zhao P: LPSinduced proinflammatory cytokine expression in human airway epithelial cells and macrophages via NFkappaB, STAT3 or AP1 activation. Mol Med Rep 17: 5484-5491, 2018.

This work is licensed under a Creative Commons Attribution-NonCommercial-NoDerivatives 4.0 International (CC BY-NC-ND 4.0) License. 